Pacific

Journal of

Mathematics

\title{
FINITE-DIMENSIONAL REPRESENTATIONS OF HYPER LOOP ALGEBRAS
}

DiJANa JAKELIĆ AND AdRiano AdREga DE Moura 


\title{
FINITE-DIMENSIONAL REPRESENTATIONS OF HYPER LOOP ALGEBRAS
}

\author{
DiJAna JAKElić AND AdRiano AdRega De Moura
}

\begin{abstract}
We study finite-dimensional representations of hyper loop algebras, that is, the hyperalgebras over an algebraically closed field of positive characteristic associated to the loop algebra over a complex finite-dimensional simple Lie algebra. The main results are the classification of the irreducible modules, a version of Steinberg's tensor product theorem, and the construction of positive characteristic analogues of the Weyl modules as defined by Chari and Pressley in the characteristic zero setting. Furthermore, we start the study of reduction modulo $p$ and prove that every irreducible module of a hyper loop algebra can be constructed as a quotient of a module obtained by a certain reduction modulo $p$ process applied to a suitable characteristic zero module. We conjecture that the Weyl modules are also obtained by reduction modulo $p$. The conjecture implies a tensor product decomposition for the Weyl modules which we use to describe the blocks of the underlying abelian category.
\end{abstract}

\section{Introduction}

Let $G$ be a semisimple connected algebraic group over an algebraically closed field $\mathbb{F}$. One can associate to $G$ its Lie algebra $L(G)$ and its algebra of distributions $U(G)$, which we prefer to call the hyperalgebra of $G$. If $\mathbb{F}$ is of characteristic zero, the hyperalgebra coincides with the universal enveloping algebra $U(L(G))$ of $L(G)$, but this is not so in positive characteristic. $U(G)$ acts naturally on any $G$-module, and it turns out that, as conjectured originally by Verma and proved by Sullivan [1978], every finite-dimensional $U(G)$-module can be "lifted" to a rational finite-dimensional $G$-module. We will restrict our attention to the case when $G$ is the Chevalley group of adjoint type associated to a complex finite-dimensional simple Lie algebra $\mathfrak{g}$. In this case the algebra $U(G)$ is isomorphic to the algebra $U(\mathfrak{g})_{\mathbb{F}}$ constructed by considering Kostant's integral form of $U(\mathfrak{g})$ and tensoring with $\mathbb{F}$ over $\mathbb{Z}$. It will suffice, for our purposes, to work over the purely algebraic setting of $U(\mathfrak{g})_{\mathbb{F}}$.

MSC2000: primary 17B10, 17B65; secondary $20 \mathrm{G} 42$.

Keywords: loop algebras, finite-dimensional representations, hyperalgebras. 
Let $\mathfrak{g}$ be as above, and let $\tilde{\mathfrak{g}}=\mathfrak{g} \otimes \mathbb{C}\left[t, t^{-1}\right]$ be the loop algebra over $\mathfrak{g}$. The finite-dimensional representation theory of $\tilde{\mathfrak{g}}$ has been a very active research topic in recent decades. It is related, for instance, to integrable models and the Bethe ansatz in statistical mechanics. Garland [1978] introduced an integral form of $U(\tilde{\mathfrak{g}})$ which can be used to construct what we call the hyper loop algebra $U(\tilde{\mathfrak{g}})_{\mathbb{F}}$ of $\mathfrak{g}$ over $\mathbb{F}$; see also [Tits 1980-1981; Mitzman 1985]. The hyperalgebra $U(\mathfrak{g})_{\mathbb{F}}$ is naturally a subalgebra of $U(\tilde{\mathfrak{g}})_{\mathbb{F}}$.

This paper studies some basic aspects of the category $\tilde{\mathscr{C}}_{\mathbb{F}}$ of finite-dimensional $U(\tilde{\mathfrak{g}})_{\mathbb{E}}$-modules, such as the classification of its simple objects and its block decomposition when $\mathbb{F}$ is an algebraically closed field of positive characteristic. In the case $\mathbb{F}=\mathbb{C}$ and thus $U(\tilde{\mathfrak{g}})_{\mathbb{F}}=U(\tilde{\mathfrak{g}})$, these questions were studied in [Chari 1986; Chari and Moura 2004; Chari and Pressley 1986]. It turns out that the simple finitedimensional $\tilde{\mathfrak{g}}$-modules are highest-weight modules with respect to the triangular decomposition of $\tilde{\mathfrak{g}}$ obtained by "looping" the usual triangular decomposition of $\mathfrak{g}$. As usual, we will call them $\ell$-highest-weight modules to distinguish them from those that are highest weight with respect to the triangular decomposition coming from the Chevalley generators of $\tilde{\mathfrak{g}}$ (nontrivial highest-weight representations with respect to the latter decomposition are infinite-dimensional). Moreover, all the simple modules are isomorphic to suitable tensor products of the so-called evaluation representations (obtained by pulling back the simple $\mathfrak{g}$-modules by the evaluation map $t \mapsto a$ for some nonzero $a \in \mathbb{C}$ ). We prove that these two results hold in positive characteristic as well. This is done in Corollary 3.2 and Theorem 3.4, the latter being a $U(\tilde{\mathfrak{g}})_{\mathbb{F}}$-version of Steinberg's tensor product theorem. Using the tensor product theorem we compute the dual representation of a given irreducible one. For highest-weight representations with respect to the usual triangular decomposition in positive characteristic, see [Garland 1978; 1980; Mathieu 1988; 1996; Tits 1987] and references therein.

The set of $\ell$-highest weights can be identified with $\operatorname{rank}(\mathfrak{g})$-tuples of polynomials in $\mathbb{F}[u]$ with constant term 1 . For $\mathbb{F}=\mathbb{C}$, Chari and Pressley [2001] showed there exists a family of universal finite-dimensional $\ell$-highest-weight modules, called the Weyl modules. We prove that the Weyl modules for $U(\tilde{\mathfrak{g}})_{\mathbb{F}}$ can be defined in a similar fashion when $\mathbb{F}$ is of positive characteristic. The reason for calling these $\ell$-highest-weight modules Weyl modules comes from a conjecture in [Chari and Pressley 2001] stating that the Weyl modules for $U(\tilde{\mathfrak{g}})$ can be obtained as the classical limit of certain irreducible finite-dimensional modules for the corresponding quantum loop algebra; the limit process resembles the one for obtaining the Weyl modules for $U(\mathfrak{g})_{\mathbb{F}}$ by reduction modulo $p$ of simple $\mathfrak{g}$-modules. Chari and Loktev [2006] proved this conjecture when $\mathfrak{g}$ is of type $A$ by using Gelfand-Tsetlin filtrations, and Fourier and Littelmann [2006] proved it when $\mathfrak{g}$ is of type $A D E$ by using Demazure modules. Moreover, H. Nakajima has pointed out that the general 
case can be deduced using the crystal and global basis results from [Beck and Nakajima 2004; Kashiwara 1994; 2002; Nakajima 2001; 2004]. Other interesting related references include [Chari and Greenstein 2007; Fourier and Littelmann 2007; Kuniba et al. 1998; 2000]. Conjecture 4.7(a) of this paper is an analogous conjecture for the Weyl modules for $U(\tilde{\mathfrak{g}})_{\mathbb{F}}$ when $\mathbb{F}$ is of positive characteristic; it states that they can be obtained from the Weyl modules for $U(\tilde{\mathfrak{g}})_{\mathbb{F}^{0}}$ by reduction modulo $p$, where $\mathbb{F}^{0}$ is a suitable field of characteristic zero. As $\mathbb{Z}$-lattices are easily seen not to be well behaved with respect to evaluation maps, we consider more general lattices for this purpose. Namely, we consider lattices over the ring $\mathbb{A}$ of Witt vectors with coefficients in $\mathbb{E}$, after changing scalars from $\mathbb{C}$ to the fraction field $\mathbb{F}^{0}$ of $\mathbb{A}$. We consider finite-dimensional $\ell$-highest-weight $U(\tilde{\mathfrak{g}})_{\mathbb{F}^{0}}$-modules such that the coefficients of the $\ell$-highest weights are in $\mathbb{A}$ and that the leading ones are units in $\mathbb{A}$; we show all such modules contain an (admissible) $\mathbb{A}$-ample-lattice. Thus, we obtain all of the irreducible modules as quotients of modules coming from a reduction modulo $p$ process. This is done in Theorem 4.5 and Corollary 4.6. Combining Conjecture 4.7 with the one in [Chari and Pressley 2001], which is now a theorem as remarked above, we have a bridge connecting the Weyl modules for $U(\tilde{\mathfrak{g}})_{\mathbb{F}}$ with certain irreducible representations for quantum loop algebras (at generic quantization parameter).

As corollaries of Conjecture 4.7, we obtain a tensor product decomposition of the Weyl modules and the block decomposition of $\widetilde{\mathscr{C}}_{\mathbb{F}}$. Although this tensor product decomposition is the natural analogue of the one obtained in [Chari and Pressley 2001] for characteristic zero, the techniques used in that paper do not seem to apply to our setting. In fact, our motivation for considering the theory of reduction modulo $p$ originated from the search for other methods which would lead to a proof of this tensor product decomposition. Indeed Conjecture 4.7(b) says that we expect that this decomposition holds in the context of A-lattices, thus transferring the problem to a characteristic zero setting. The block decomposition of $\tilde{\mathscr{C}}_{\mathbb{F}}$ is described similarly to that of $\tilde{\mathscr{C}}_{\mathbb{C}}$ as well, that is, the blocks are parametrized by functions with finite support $\chi: \mathbb{F}^{\times} \rightarrow P / Q$ called spectral characters. Here $P$ and $Q$ are the weight and root lattices of $\mathfrak{g}$, and $\mathbb{F}^{\times}=\mathbb{F}-\{0\}$. The proof runs parallel to its characteristic zero counterpart found in [Chari and Moura 2004]; hence, the tensor product decomposition for Weyl modules plays a key role. However, our results on reduction modulo $p$ are needed both to prove that the Weyl modules have well-defined spectral characters and to obtain a positive characteristic version of [Chari and Moura 2004, Proposition 3.4] - a key ingredient in the construction of certain useful indecomposable modules.

In Section 1, we fix the basic notation for finite-dimensional complex simple Lie algebras and their loop algebras, define the hyperalgebras, and collect some important lemmas. Section 2 reviews the relevant facts about finite-dimensional 
$U(\mathfrak{g})_{\mathbb{F}}$-modules. Sections 3 and 4 comprise the main part of the paper. In Section $3 \mathrm{~A}$, we define $\ell$-highest-weight modules and obtain the necessary relations satisfied by the finite-dimensional ones. Section 3B classifies the irreducible modules and arrives at the aforementioned tensor product and duality results. Section 3C constructs the Weyl modules. Section 4 ends the paper with the results and the conjecture on reduction modulo $p$ as well as their application to describing the blocks.

\section{Hyperalgebras}

Throughout, $\mathbb{C}, \mathbb{Z}, \mathbb{Z}_{+}$, and $\mathbb{N}$ denote the sets of complex numbers, integers, nonnegative integers, and positive integers. For a ring $\mathbb{A}$, we denote the underlying multiplicative group of units by $\mathbb{A}^{\times}$. The dual of a vector space $V$ is $V^{*}$.

1A. Preliminaries. Let $I$ be the set of vertices of a finite-type connected Dynkin diagram, and let $\mathfrak{g}$ be the associated simple complex Lie algebra with a fixed Cartan subalgebra $\mathfrak{h}$ and nilpotent subalgebras $\mathfrak{n}^{ \pm}$. Denote by $R^{+}$the set of positive roots such that

$$
\mathfrak{n}^{ \pm}=\bigoplus_{\alpha \in R^{+}} \mathfrak{g}_{ \pm \alpha}, \quad \text { where } \mathfrak{g}_{ \pm \alpha}=\{x \in \mathfrak{g}:[h, x]= \pm \alpha(h) x, \text { for all } h \in \mathfrak{h}\} .
$$

We denote the simple roots by $\alpha_{i}$, the fundamental weights by $\omega_{i}$, and by $Q, Q^{+}$ and $P, P^{+}$the root and weight lattices with corresponding positive cones. We equip $\mathfrak{h}^{*}$ with the partial order $\lambda \leq \mu$ if and only if $\mu-\lambda \in Q^{+}$. We denote the Weyl group by $\mathcal{W}$, its longest element by $w_{0}$, and the maximal positive root by $\theta$. Let $\langle\cdot, \cdot\rangle$ be the bilinear form on $\mathfrak{h}^{*}$ induced by the Killing form on $\mathfrak{g}$, and for $\lambda \in \mathfrak{h}^{*}-\{0\}$, set $\lambda^{\vee}=2 \lambda /\langle\lambda, \lambda\rangle$ and $d_{\lambda}=\frac{1}{2}\langle\lambda, \lambda\rangle$. Then $\left\{\alpha_{i}^{\vee}: i \in I\right\}$ is a set of simple roots of the simple Lie algebra $\mathfrak{g}^{\vee}$ whose Dynking diagram is obtained from that of $\mathfrak{g}$ by reversing the arrows, and $R^{\vee}=\left\{\alpha^{\vee}: \alpha \in R\right\}$ is its root system, where $R=R^{+} \cup\left(-R^{+}\right)$. If $\alpha=\sum_{i} m_{i} \alpha_{i}$ and $\alpha^{\vee}=\sum_{i} m_{i}^{\vee} \alpha_{i}^{\vee}$, then

$$
m_{i}^{\vee}=\frac{d_{\alpha_{i}}}{d_{\alpha}} m_{i}
$$

If $\mathfrak{a}$ is a Lie algebra (over any field $\mathbb{F}$ ), define its loop algebra $\tilde{\mathfrak{a}}=\mathfrak{a} \otimes_{\mathbb{F}} \mathbb{F}\left[t, t^{-1}\right]$ with bracket $\left[x \otimes t^{r}, y \otimes t^{s}\right]=[x, y] \otimes t^{r+s}$. Clearly $\mathfrak{a} \otimes 1$ is a subalgebra of $\tilde{\mathfrak{a}}$ isomorphic to $\mathfrak{a}$, and by abuse of notation, we will continue denoting its elements by $x$ instead of $x \otimes 1$. In case $\mathfrak{a}=\mathfrak{g}$, we have $\tilde{\mathfrak{g}}=\tilde{\mathfrak{n}}^{-} \oplus \tilde{\mathfrak{h}} \oplus \tilde{\mathfrak{n}}^{+}$, and $\tilde{\mathfrak{h}}$ is an abelian subalgebra.

Let $U(\mathfrak{a})$ be the universal enveloping algebra of $\mathfrak{a}$. Then $U(\mathfrak{a})$ is a subalgebra of $U(\tilde{\mathfrak{a}})$, and for $\mathfrak{a}=\mathfrak{g}$, multiplication establishes isomorphisms

$$
U(\mathfrak{g}) \cong U\left(\mathfrak{n}^{-}\right) \otimes U(\mathfrak{h}) \otimes U\left(\mathfrak{n}^{+}\right) \quad \text { and } \quad U(\tilde{\mathfrak{g}}) \cong U\left(\tilde{\mathfrak{n}}^{-}\right) \otimes U(\tilde{\mathfrak{h}}) \otimes U\left(\tilde{\mathfrak{n}}^{+}\right) .
$$


The assignments

$$
\begin{aligned}
\triangle: \mathfrak{a} & \rightarrow U(\mathfrak{a}) \otimes_{\mathbb{F}} U(\mathfrak{a}), x \mapsto x \otimes 1+1 \otimes x, \\
S: \mathfrak{a} & \rightarrow \mathfrak{a}, x \mapsto-x, \\
\epsilon: \mathfrak{a} & \rightarrow \mathbb{F}, x \mapsto 0
\end{aligned}
$$

can be uniquely extended so that $U(\mathfrak{a})$ becomes a Hopf algebra with comultiplication $\triangle$, antipode $S$, and counit $\epsilon$. We shall denote by $U(\mathfrak{a})^{0}$ the augmentation ideal, that is, the kernel of $\epsilon$. Consider the associative $\mathbb{F}$-algebra $U(\mathfrak{a}) \otimes_{\mathbb{F}} \mathbb{F}\left[t, t^{-1}\right]$ with the obvious tensor product structure and the usual bracket. Clearly the inclusion $\tilde{\mathfrak{a}} \hookrightarrow U(\mathfrak{a}) \otimes_{\mathbb{F}} \mathbb{F}\left[t, t^{-1}\right]$ is a Lie algebra map. Therefore the next lemma is immediate from the universal property of $U(\tilde{\mathfrak{a}})$.

Lemma 1.1. There exists a unique algebra map $U(\tilde{\mathfrak{a}}) \rightarrow U(\mathfrak{a}) \otimes_{\mathbb{F}} \mathbb{E}\left[t, t^{-1}\right]$ which is the identity on $\tilde{\mathfrak{a}}$.

We call this map the formal evaluation map and denote it by ev. For each $a \in \mathbb{F}^{\times}$, consider the evaluation map $U(\mathfrak{a}) \otimes_{\mathbb{F}} \mathbb{E}\left[t, t^{-1}\right] \rightarrow U(\mathfrak{a})$ sending $x \otimes f(t)$ to $f(a) x$, and denote by $\mathrm{ev}_{a}$ the composition of this map with ev. Then $\mathrm{ev}_{a}$ is a surjective algebra homomorphism ev $\mathrm{ev}_{a}: U(\tilde{\mathfrak{a}}) \rightarrow U(\mathfrak{a})$ which we call the evaluation map at $a$.

Remark. Obviously, an existence proof for $\mathrm{ev}_{a}$ could be constructed like that of ev. However, we will use the formal evaluation map to prove the existence of evaluation maps in the context of hyper loop algebras using $\mathbb{Z}$-lattices only; see Proposition 3.3 and the remark after Proposition 4.10.

1B. Reduction modulo $p$. As usual, given any associative algebra $A$ over a field of characteristic zero, $a \in A$, and $k \in \mathbb{Z}_{+}$, we set

$$
a^{(k)}=\frac{a^{k}}{k !} \quad \text { and } \quad\left(\begin{array}{l}
a \\
k
\end{array}\right)=\frac{a(a-1) \cdots(a-k+1)}{k !} \in A .
$$

Let $\Phi=\left\{x_{\alpha}^{ \pm}, h_{\alpha_{i}}: \alpha \in R^{+}, i \in I\right\}$ be a Chevalley basis for $\mathfrak{g}$, where $x_{\alpha}^{ \pm} \in \mathfrak{g}_{ \pm \alpha}$. Set $h_{\alpha}=\left[x_{\alpha}^{+}, x_{\alpha}^{-}\right], x_{\alpha, r}^{ \pm}=x_{\alpha}^{ \pm} \otimes t^{r}$, and $h_{\alpha, r}=h_{\alpha} \otimes t^{r}$. When $r=0$ we may just write $x_{\alpha}^{ \pm}$and $h_{\alpha}$. Also, we may write $x_{i, r}^{ \pm}$and $h_{i, r}$ in place of $x_{\alpha_{i}, r}^{ \pm}$and $h_{\alpha_{i}, r}$. Note the set $\tilde{\Phi}=\left\{x_{\alpha, r}^{ \pm}, h_{i, r}: \alpha \in R^{+}, i \in I, r \in \mathbb{Z}\right\}$ is a basis for $\tilde{\mathfrak{g}}$, and define $\tilde{\mathfrak{g}}_{\mathbb{Z}}$ to be the $\mathbb{Z}$-span of $\tilde{\Phi}$. The $\mathbb{Z}$-span of $\Phi$ is a Lie $\mathbb{Z}$-subalgebra of $\tilde{\mathfrak{g}}_{\mathbb{Z}}$ which we denote by $\mathfrak{g}_{\mathbb{Z}}$.

If $\mathbb{F}$ is any field, set $\mathfrak{g}_{\mathbb{F}}=\mathfrak{g}_{\mathbb{Z}} \otimes_{\mathbb{Z}} \mathbb{F}$ and $\tilde{\mathfrak{g}}_{\mathbb{F}}=\tilde{\mathfrak{g}}_{\mathbb{Z}} \otimes_{\mathbb{Z}} \mathbb{F}$, so that $\mathfrak{g}_{\mathbb{F}}$ and $\tilde{\mathfrak{g}}_{\mathbb{F}}$ are Lie algebras over $\mathbb{F}$. 
Given $\alpha \in R^{+}$and $r \in \mathbb{Z}$, define elements $\Lambda_{\alpha, r} \in U(\tilde{\mathfrak{h}})$ by equating the following formal power series in $u$ :

$$
\Lambda_{\alpha}^{ \pm}(u)=\sum_{r=0}^{\infty} \Lambda_{\alpha, \pm r} u^{r}=\exp \left(-\sum_{s=1}^{\infty} \frac{h_{\alpha, \pm s}}{s} u^{s}\right) .
$$

We may write $\Lambda_{i, r}$ for $\Lambda_{\alpha_{i}, r}$. It follows from (1-1) that, if $\alpha=\sum_{i} m_{i} \alpha_{i} \in R^{+}$, then $h_{\alpha}=\sum_{i} m_{i}^{\vee} h_{i}$ and

$$
\Lambda_{\alpha}^{ \pm}(u)=\prod_{i \in I}\left(\Lambda_{\alpha_{i}}^{ \pm}(u)\right)^{m_{i}^{\vee}} .
$$

We have [Garland 1978, Lemma 5.1]:

$$
\operatorname{ev}\left(\Lambda_{\alpha, r}\right)=(-1)^{r}\left(\begin{array}{c}
h_{\alpha} \\
|r|
\end{array}\right) \otimes t^{r}
$$

Set

$$
H_{\alpha}(u)=\operatorname{ev}_{-1}\left(\Lambda_{\alpha}^{+}(u)\right)=\exp \left(-\sum_{s \geq 1} h_{\alpha} \frac{(-u)^{s}}{s}\right),
$$

so that $\left(H_{\alpha}(u)\right)_{k}$, that is, the coefficient of $u^{k}$ in $H_{\alpha}(u)$, is $\left(\begin{array}{c}h_{\alpha} \\ k\end{array}\right)$.

For $k \in \mathbb{Z}$ with $k \neq 0$, consider also the endomorphism $\tau_{k}$ of $U(\tilde{\mathfrak{g}})$ extending $t \mapsto t^{k}$, and set $\Lambda_{\alpha, r ; k}=\tau_{k}\left(\Lambda_{\alpha, r}\right)$ and $\Lambda_{\alpha ; k}^{ \pm}(u)=\sum_{r=0}^{\infty} \Lambda_{\alpha, \pm r ; k} u^{r}$. Notice that $\left(\begin{array}{c}h_{i} \\ k\end{array}\right)$ is a polynomial in $h_{i}$ of degree $k$. Hence the set

$$
\left\{\left(\begin{array}{l}
h_{1} \\
k_{1}
\end{array}\right) \cdots\left(\begin{array}{l}
h_{\ell} \\
k_{\ell}
\end{array}\right): k_{j} \in \mathbb{Z}_{+}\right\}, \quad \text { where } \ell=|I|,
$$

is a basis for $U(\mathfrak{h})$. Similarly, observe that $\Lambda_{i, \pm r ; k}$ for $r, k \in \mathbb{N}$ is a polynomial in $h_{i, \pm s k}$ with $1 \leq s \leq r$ whose leading term is $\left(-h_{i, \pm k}\right)^{(r)}$. Finally, given an order on $\tilde{\Phi}$ and a PBW monomial with respect to this order, we construct an ordered monomial in the elements

$$
\left(x_{\alpha, r}^{ \pm}\right)^{(k)}, \quad \Lambda_{i, r ; k}, \quad\left(\begin{array}{c}
h_{i} \\
k
\end{array}\right) \text { for } r, k \in \mathbb{Z} \text { with } k>0, \alpha \in R^{+}, \text {and } i \in I
$$

using the correspondence just discussed for the basis elements of $U(\tilde{\mathfrak{h}})$, as well as the obvious correspondence $\left(x_{\alpha, r}^{ \pm}\right)^{k} \leftrightarrow\left(x_{\alpha, r}^{ \pm}\right)^{(k)}$. The set of monomials thus obtained is then a basis for $U(\tilde{\mathfrak{g}})$, while the monomials involving only $\left(x_{\alpha}^{ \pm}\right)^{(k)}$ and $\left(\begin{array}{c}h_{i} \\ k\end{array}\right)$ form a basis for $U(\mathfrak{g})$. Let $U(\tilde{\mathfrak{g}})_{\mathbb{Z}}$ and $U(\mathfrak{g})_{\mathbb{Z}}$ be the respective $\mathbb{Z}$-spans of these monomials. The following theorems are crucial:

Theorem 1.2 [Kostant 1966]. $U(\mathfrak{g})_{\mathbb{Z}}$ is the $\mathbb{Z}$-subalgebra of $U(\mathfrak{g})$ generated by $\left\{\left(x_{\alpha}^{ \pm}\right)^{(k)}: \alpha \in R^{+}\right.$and $\left.k \in \mathbb{Z}_{+}\right\}$.

Theorem 1.3 [Garland 1978]. $U(\tilde{\mathfrak{g}})_{\mathbb{Z}}$ is the $\mathbb{Z}$-subalgebra of $U(\tilde{\mathfrak{g}})$ generated by $\left\{\left(x_{\alpha, r}^{ \pm}\right)^{(k)}: \alpha \in R^{+}\right.$and $r, k \in \mathbb{Z}$ with $\left.k \geq 0\right\}$. 
For $\mathfrak{a} \in\left\{\mathfrak{g}, \mathfrak{n}^{ \pm}, \mathfrak{h}, \tilde{\mathfrak{g}}, \tilde{\mathfrak{n}}^{ \pm}, \tilde{\mathfrak{h}}\right\}$, let $U(\mathfrak{a})_{\mathbb{Z}}$ denote the corresponding $\mathbb{Z}$-subalgebra of $U(\tilde{\mathfrak{g}})$. Given a field $\mathbb{F}$, the $\mathbb{F}$-hyperalgebra of $\mathfrak{a}$ is defined by

$$
U(\mathfrak{a})_{\mathbb{F}}=U(\mathfrak{a})_{\mathbb{Z}} \otimes_{\mathbb{Z}} \mathbb{F} .
$$

We will also refer to $U(\tilde{\mathfrak{g}})_{\mathbb{F}}$ as the hyper loop algebra of $\mathfrak{g}$ over $\mathbb{F}$. The PBW Theorem gives

$$
U(\mathfrak{g})_{\mathbb{F}}=U\left(\mathfrak{n}^{-}\right)_{\mathbb{F}} U(\mathfrak{h})_{\mathbb{E}} U\left(\mathfrak{n}^{+}\right)_{\mathbb{F}} \quad \text { and } \quad U(\tilde{\mathfrak{g}})_{\mathbb{F}}=U\left(\tilde{\mathfrak{n}}^{-}\right)_{\mathbb{F}} U(\tilde{\mathfrak{h}})_{\mathbb{F}} U\left(\tilde{\mathfrak{n}}^{+}\right)_{\mathbb{F}} .
$$

Clearly, if $\mathbb{F}$ is of characteristic zero, $U(\mathfrak{a})_{\mathbb{F}}$ is naturally isomorphic to $U\left(\mathfrak{a}_{\mathbb{F}}\right)$. For fields of positive characteristic we just have an algebra homomorphism $U\left(\mathfrak{a}_{\mathbb{F}}\right) \rightarrow$ $U(\mathfrak{a})_{\mathbb{F}}$, which is neither injective nor surjective. If no confusion arises, we will write $x$ instead of $x \otimes 1$ for the image of an element $x \in U(\tilde{\mathfrak{g}})_{\mathbb{Z}}$ in $U(\tilde{\mathfrak{g}})_{\mathbb{F}}$.

Quite clearly the Hopf algebra structure on $U(\tilde{\mathfrak{g}})$ preserves the $\mathbb{Z}$-forms $U(\mathfrak{a})_{\mathbb{Z}}$ and therefore induces a Hopf algebra structure on $U(\tilde{\mathfrak{g}})_{\mathbb{F}}$ with counit given by $\epsilon\left(\left(x_{\alpha, r}^{ \pm}\right)^{(k)}\right)=0$ and comultiplication determined by

$$
\begin{aligned}
\triangle\left(\left(x_{\alpha, r}^{ \pm}\right)^{(k)}\right) & =\sum_{l+m=k}\left(x_{\alpha, r}^{ \pm}\right)^{(l)} \otimes\left(x_{\alpha, r}^{ \pm}\right)^{(m)}, \\
\triangle\left(\left(\begin{array}{c}
h_{i} \\
k
\end{array}\right)\right) & =\sum_{l+m=k}\left(\begin{array}{c}
h_{i} \\
l
\end{array}\right) \otimes\left(\begin{array}{c}
h_{i} \\
m
\end{array}\right), \\
\triangle\left(\Lambda_{\alpha, \pm k}\right) & =\sum_{l+m=k} \Lambda_{\alpha, \pm l} \otimes \Lambda_{\alpha, \pm m} .
\end{aligned}
$$

Moreover, the antipode on the basis of $U(\tilde{\mathfrak{h}})_{\mathbb{F}}$ is determined by

$$
S\left(\Lambda_{\alpha ; k}^{ \pm}(u)\right)=\left(\Lambda_{\alpha ; k}^{ \pm}(u)\right)^{-1} \quad \text { and } \quad S\left(H_{\alpha}(u)\right)=\left(H_{\alpha}(u)\right)^{-1},
$$

where the inverses in the last two equations are the ones of formal power series.

1C. Some lemmas. We now collect some essential identities on $U(\tilde{\mathfrak{g}})_{\mathbb{F}}$, when $\mathbb{F}$ is a field of characteristic $p>0$. We begin with the trivial observation that

$$
\left(x_{\alpha, r}^{ \pm}\right)^{(k)}\left(x_{\alpha, r}^{ \pm}\right)^{(l)}=\left(\begin{array}{c}
k+l \\
k
\end{array}\right)\left(x_{\alpha, r}^{ \pm}\right)^{(k+l)} .
$$

From this, one easily deduces

$$
\left(\left(x_{\alpha, r}^{ \pm}\right)^{(k)}\right)^{p}=0 .
$$

It is well known [Humphreys 1977] that the elements $\left(\begin{array}{c}h_{i} \\ k\end{array}\right)$ satisfy

$$
\left(\begin{array}{c}
h_{i} \\
k
\end{array}\right)^{p}=\left(\begin{array}{c}
h_{i} \\
k
\end{array}\right),
$$


and it is easy to see that

$$
\left(\begin{array}{c}
h_{i} \\
l
\end{array}\right)\left(x_{\alpha, r}^{ \pm}\right)^{(k)}=\left(x_{\alpha, r}^{ \pm}\right)^{(k)}\left(\begin{array}{c}
h_{i} \pm k \alpha\left(h_{i}\right) \\
l
\end{array}\right) .
$$

Given $\alpha \in R^{+}$and $s \in \mathbb{Z}$, define

$$
X_{\alpha ; s, \pm}^{-}(u)=\sum_{r \geq 1} x_{\alpha, \pm(r+s)}^{-} u^{r} .
$$

Lemma 1.4. We have

$$
\left(x_{\alpha}^{+}\right)^{(l)}\left(x_{\alpha}^{-}\right)^{(k)}=\sum_{m=0}^{\min \{k, l\}}\left(x_{\alpha}^{-}\right)^{(k-m)}\left(\begin{array}{c}
h_{\alpha}-k-l+2 m \\
m
\end{array}\right)\left(x_{\alpha}^{+}\right)^{(l-m)}
$$

and

$$
\left(x_{\alpha, \mp s}^{+}\right)^{(l)}\left(x_{\alpha, \pm(s+1)}^{-}\right)^{(k)} \in(-1)^{l}\left(\left(X_{\alpha ; s, \pm}^{-}(u)\right)^{(k-l)} \Lambda_{\alpha}^{ \pm}(u)\right)_{k}+U(\tilde{\mathfrak{g}})_{\mathbb{E}} U\left(\tilde{\mathfrak{n}}^{+}\right)_{\mathbb{F}}^{0} .
$$

In (1-11), $k \geq l \geq 1$, and the subindex $k$ means the coefficient of $u^{k}$ of the power series above.

Proof. It suffices to prove that the relations hold in $U(\tilde{\mathfrak{g}})_{\mathbb{Z}}$. For both claims, the strategy is to commute the elements on the left hand side. The proof of (1-10) can be found in [Humphreys 1972, Lemma 26.2].

Relation (1-11) was proved in [Garland 1978, Lemma 7.5] for $s=0$ and the choice of " \pm " such that we have " + " on the right hand side. Consider the subalgebra of $U(\tilde{\mathfrak{g}})_{\mathbb{Z}}$ generated by $\left(x_{\alpha, r}^{ \pm}\right)^{(k)}$ for a fixed $\alpha \in R^{+}$. It is easy to see that, for each $s \in \mathbb{Z}$, the assignment $\left(x_{\alpha, r}^{ \pm}\right)^{(k)} \mapsto\left(x_{\alpha, r \pm s}^{ \pm}\right)^{(k)}$ extends uniquely to an algebra automorphism of this subalgebra which is the identity when restricted to $U(\tilde{\mathfrak{h}})_{\mathbb{Z}}$. The general case of (1-11) (with "+" on the right hand side) follows easily from the case $s=0$ using these automorphisms; see also [Chari and Pressley 2001, Lemma 1.3]. For the opposite choice of " \pm ", just apply the automorphism determined by the assignment $\left(x_{\alpha, r}^{ \pm}\right)^{(k)} \mapsto\left(x_{\alpha,-r}^{ \pm}\right)^{(k)}$.

The following lemmas will be needed in the proof of Theorem 3.11. Consider monomials involving only the elements $\left(x_{\alpha, r}^{-}\right)^{(k)}$. Define the degree of $\left(x_{\alpha, r}^{-}\right)^{(k)}$ to be $k$, and extend it additively.

Lemma 1.5. Let $\alpha, \beta \in R^{+}, k, l \in \mathbb{Z}_{+}$, and $r, s \in \mathbb{Z}$. Then $\left(x_{\alpha, r}^{-}\right)^{(k)}\left(x_{\beta, s}^{-}\right)^{(l)}$ is in the span of $\left(x_{\beta, s}^{-}\right)^{(l)}\left(x_{\alpha, r}^{-}\right)^{(k)}$ together with monomials of degree strictly smaller than $k+l$.

Proof. Using that $U\left(\tilde{\mathfrak{n}}^{-}\right)_{\mathbb{Z}}$ is $\left(Q^{+} \times \mathbb{Z}\right)$-graded, the claim follows immediately from the proof of [Humphreys 1972, Lemma 26.3.C]. 
The next lemma is part of [Garland 1978, Lemma 5.11] and shows that the elements $\Lambda_{\alpha, r ; k}$ are linear combinations of products of the elements $\Lambda_{\alpha, s}$.

Lemma 1.6. In $U(\tilde{\mathfrak{h}})_{\mathbb{Z}}$, for all $k, s \in \mathbb{N}$ and $\alpha \in R^{+}$, we have

$$
\Lambda_{\alpha, \pm s ; k}=k \Lambda_{\alpha, \pm s k}+\sum_{(\vec{r}, \vec{n})} m_{\vec{r}, \vec{n}} \Lambda_{\alpha, \pm r_{1}}^{n_{1}} \cdots \Lambda_{\alpha, \pm r_{l}}^{n_{l}} \quad \text { for some } m_{\vec{r}, \vec{n}} \in \mathbb{Z}
$$

The sum is over the pairs $(\vec{r}, \vec{n})$ where $\vec{r}=\left(r_{1}, \ldots, r_{l}\right)$ and $\vec{n}=\left(n_{1}, \ldots, n_{l}\right)$ are such that $l, r_{j}, n_{j} \in \mathbb{N}, r_{i} \neq r_{j}, l \sum_{j} n_{j}>1$, and $\sum_{j} n_{j} r_{j}=s k$.

\section{D. Frobenius homomorphism.}

Lemma 1.7. $U(\tilde{\mathfrak{g}})_{\mathbb{F}}$ is generated as an algebra by the elements $\left(x_{\alpha, r}^{ \pm}\right)^{\left(p^{k}\right)}$, and $U(\mathfrak{g})_{\mathbb{F}}$ is generated by $\left(x_{\alpha}^{ \pm}\right)^{\left(p^{k}\right)}$, where, in both statements, $\alpha \in R^{+}, k, r \in \mathbb{Z}$, and $k \geq 0$. Moreover, $U(\mathfrak{h})_{\mathbb{F}}$ is generated as an algebra by $\left(\begin{array}{c}h_{i} \\ p^{k}\end{array}\right)$ for $i \in I$ and $k \in \mathbb{Z}_{+}$.

Proof. The first two statements are immediate from Theorems Theorem 1.2 and 1.3 together with (1-7). The last is a statement on $U(\mathfrak{g})_{\mathbb{F}}$ and is well known.

It is known that there exists a Hopf algebra map $\tilde{\phi}: U(\tilde{\mathfrak{g}})_{\mathbb{F}} \rightarrow U(\tilde{\mathfrak{g}})_{\mathbb{F}}$ sending $\left(x_{\alpha, r}^{ \pm}\right)^{\left(p^{k}\right)}$ to $\left(x_{\alpha, r}^{ \pm}\right)^{\left(p^{k-1}\right)}$, with the convention that the latter is zero when $k=0$. We will denote the restriction of $\tilde{\phi}$ to $U(\mathfrak{g})_{\mathbb{F}}$ by $\phi$ and call both of them the (arithmetic) Frobenius homomorphisms. The first formula below is well known and the second was proved in [Cline et al. 2000].

$$
\phi\left(\left(\begin{array}{c}
h_{i} \\
p^{k}
\end{array}\right)\right)=\left(\begin{array}{c}
h_{i} \\
p^{k-1}
\end{array}\right) \quad \text { and } \quad \tilde{\phi}\left(\Lambda_{i, r}\right)= \begin{cases}\Lambda_{i, r / p} & \text { if } p \text { divides } r \\
0 & \text { otherwise. }\end{cases}
$$

The proof of the existence of the map $\phi$ can be found in [Jantzen 1987], for instance. For the existence of $\tilde{\phi}$, see [Mathieu 1996, Lemma 1.3] and [Chari and Pressley 1997, Lemma 9.5].

Given a $U(\tilde{\mathfrak{g}})_{\mathbb{F}}$-module $V$, we denote by $V^{\tilde{\phi}^{m}}$ the pullback of $V$ by $\tilde{\phi}^{m}$. Similarly if $V$ is a $U(\mathfrak{g})_{\mathbb{F}}$-module, $V^{\phi^{m}}$ will denote the pullback of $V$ by $\phi^{m}$.

\section{Review of finite-dimensional $U(\mathfrak{g})_{\mathbb{F}}$-modules}

In this section we review some results on finite-dimensional representations of $U(\mathfrak{g})_{\mathbb{F}}$. In the first subsection we consider the case $\mathbb{F}=\mathbb{C}$, where we summarize the basic results without proofs. The literature for this subsection is vast and well known; all the results we mention can be found, for example, in [Humphreys 1972]. In the other subsections $\mathbb{F}$ will be an algebraically closed field of characteristic $p>0$. Jantzen [1987] gives essentially all of the results, although the approach is heavily geometric; see also [Borel et al. 1970]. Our approach follows that of [Humphreys 1972, Chapter VII] and [Humphreys 1977]. Since some proofs are relevant for Section 3, we consider it appropriate to sketch them. 
2A. Characteristic zero and lattices. Given a $U(\mathfrak{g})$-module $V$, a vector $v \in V$ is called a weight vector if $h v=\mu(h) v$ for some $\mu \in \mathfrak{h}^{*}$ and all $h \in \mathfrak{h}$. The subspace consisting of weight vectors of weight $\mu$ will be denoted by $V_{\mu}$. If $v$ is a weight vector such that $\mathfrak{n}^{+} v=0$, then $v$ is called a highest-weight vector. If $V$ is generated by a highest-weight vector of weight $\lambda$, then $V$ is said to be a highest-weight module of highest weight $\lambda$.

Here is a summary of the basic facts of finite-dimensional $U(\mathfrak{g})$-modules:

Theorem 2.1. Let $V$ be a finite-dimensional $U(\mathfrak{g})$-module.

(a) $V=\bigoplus_{\mu \in \mathfrak{h}^{*}} V_{\mu}$ and $\operatorname{dim} V_{\mu}=\operatorname{dim} V_{w \mu}$ for all $w \in \mathscr{W}$.

(b) $V$ is completely reducible.

(c) For each $\lambda \in P^{+}$the $U(\mathfrak{g})$-module $V^{0}(\lambda)$ generated by a vector $v$ satisfying

$$
x_{i}^{+} v=0, \quad h_{i} v=\lambda\left(h_{i}\right) v, \quad \text { and }\left(x_{i}^{-}\right)^{\lambda\left(h_{i}\right)+1} v=0 \quad \text { for all } i \in I
$$

is irreducible and finite-dimensional. If $V$ is irreducible, then $V$ is isomorphic to $V^{0}(\lambda)$ for some $\lambda \in P^{+}$.

(d) If $\lambda \in P^{+}$and $V \cong V^{0}(\lambda)$, then $V_{\mu} \neq 0$ if and only if $w \mu \leq \lambda$ for all $w \in W$. Furthermore, the minimal weight of $V^{0}(\lambda)$ is $w_{0} \lambda$.

An admissible lattice for a $U(\mathfrak{g})$-module $V$ is the $\mathbb{Z}$-span of a basis for $V$ that is invariant under the action of $U(\mathfrak{g})_{\mathbb{Z}}$. The basic results about lattices are summarized next; see [Humphreys 1972].

Theorem 2.2. Let $V, W$ be finite-dimensional $U(\mathfrak{g})$-modules.

(a) If $L$ is an additive subgroup of $V$ that is invariant under the action of $U(\mathfrak{g})_{\mathbb{Z}}$, then $L=\bigoplus_{\mu \in P} L_{\mu}$, where $L_{\mu}=L \cap V_{\mu}$.

(b) There exists an admissible lattice for $V$.

(c) If $L, M$ are admissible lattices for $V, W$, respectively, then $L \otimes_{\mathbb{Z}} M$ is an admissible lattice for $V \otimes W$.

(d) If $V$ is an irreducible module and $v$ is a highest-weight vector of weight $\lambda$, then $L=U\left(\mathfrak{n}^{-}\right)_{\mathbb{Z}} v$ is minimal in the set of admissible lattices for $V$ satisfying $L_{\lambda}=\mathbb{Z} v$.

2B. Classification of irreducible modules in positive characteristic. From now on, $\mathbb{F}$ is an algebraically closed field of characteristic $p>0$, and $\mathbb{F}_{p}$ denotes its prime field. In this subsection we recall the methods used to classify the irreducible representations of $U(\mathfrak{g})_{\mathbb{F}}$ up to isomorphism. Although the classification is the same as in the case of $U(\mathfrak{g})$, the methods are quite different and will be used when we treat the case of $U(\tilde{\mathfrak{g}})_{\mathbb{F}}$. 


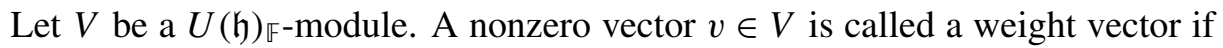
there exists $z=\left(z_{i, k}\right)$, called the weight of $v$, with $z_{i, k} \in \mathbb{F}$ and $i \in I, k \in \mathbb{Z}_{+}$, such that $\left(\begin{array}{c}h_{i} \\ p^{k}\end{array}\right) v=z_{i, k} v$. Notice that (1-8) implies $z_{i, k}$ must be in $\mathbb{F}_{p}$. We say that $z$ is integral if $z_{i, k}=\left(\begin{array}{c}\mu\left(h_{i}\right) \\ p^{k}\end{array}\right)$ for some $\mu \in P$; we say $z$ is dominant integral if the same is true for some $\mu \in P^{+}$. In those cases we identify $z$ with $\mu$ and say that $v$ has

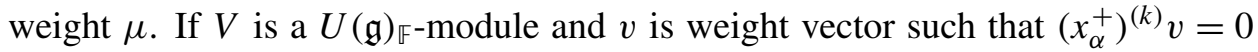
for all $\alpha \in R^{+}$and $k \in \mathbb{N}$, then $v$ is said to be a highest-weight vector. If $V$ is generated by a highest-weight vector, $V$ is called a highest-weight module.

Since the $\left(\begin{array}{l}h_{i} \\ p^{k}\end{array}\right)$ commute, we can decompose any finite-dimensional representation $V$ of $U(\mathfrak{g})_{\mathbb{F}}$ in a direct sum of generalized eigenspaces for the action of $U(\mathfrak{h})_{\mathbb{F}}$ :

$$
V=\bigoplus_{z} V_{z}
$$

We say that $z$ is a weight of $V$ if $V_{\boldsymbol{z}} \neq 0$, and in that case, $V_{\boldsymbol{z}}$ is called a weight space of $V$. When $z$ is integral we write $V_{\mu}$ instead of $V_{z}$.

Given $z=\left(z_{i, k}\right)$ and $\mu \in P$ define $z+\mu=\boldsymbol{y}$ by the equality

$$
y_{i, k} v=\left(\begin{array}{c}
h_{i}+\mu\left(h_{i}\right) \\
p^{k}
\end{array}\right) v,
$$

where $v$ is some weight vector of weight $z$. It follows from (1-9) that if $v$ has weight $z$, then $\left(x_{\alpha}^{ \pm}\right)^{(k)} v$ is either zero or has weight $z \pm k \alpha$. Hence, if $v$ is a highest-weight vector for a highest-weight representation $V$, we have $\operatorname{dim}\left(V_{\boldsymbol{z}}\right)=1$ and $V \boldsymbol{y} \neq 0$ only if $\boldsymbol{y} \leq \boldsymbol{z}$, where $\boldsymbol{y} \leq \boldsymbol{z}$ if and only if $\boldsymbol{y}=\boldsymbol{z}-\eta$ for some $\eta \in Q^{+}$. Standard arguments then show:

Proposition 2.3. Every highest-weight module is indecomposable and has a unique maximal proper submodule and hence also a unique irreducible quotient.

Any nonnegative integer $m$ can be written uniquely as $m=\sum_{j \geq 0} m_{j} p^{j}$, where $0 \leq m_{j}<p$, so that $\left(\begin{array}{c}m \\ p^{r}\end{array}\right)=m_{r}(\bmod p)$ for all $r \geq 0$. We shall write $\bar{m}$ for the image of $m \in \mathbb{Z}$ in $\mathbb{F}_{p}$.

Theorem 2.4. If $V$ is an irreducible finite-dimensional $U(\mathfrak{g})_{\mathbb{E}}$-module, then $V$ is a highest-weight representation with dominant integral highest weight.

Proof. As $V$ is irreducible, the generalized eigenspaces $V_{\boldsymbol{z}}$ are in fact eigenspaces. Since $V$ is finite-dimensional, it also follows that there exists a maximal weight $z$, and hence $V$ is a highest-weight module. It remains to prove that $z$ is dominant integral. Let $v$ be a highest-weight vector for $V$. As we observed above, $\left(x_{\alpha}^{-}\right)^{(k)} v$ is either zero or has weight $z-k \alpha$. This implies that, for every $i \in I$, there exists $N_{i} \in \mathbb{Z}_{+}$minimal such that $\left(x_{i}^{-}\right)^{\left(p^{k}\right)} v=0$ for all $k \geq N_{i}$. Moreover, we conclude from (1-10) with $k=l \geq p^{N_{i}}$ that $\left(\begin{array}{c}h_{i} \\ p^{r}\end{array}\right) v=0$ for all $r \geq N_{i}$. Now we easily see that $z$ coincides with $\lambda \in P^{+}$defined by $\lambda\left(h_{i}\right)=\sum_{j=0}^{N_{i}-1} m_{i, j} p^{j}$ with $0 \leq m_{i, j}<p$ such that $\bar{m}_{i, r}=z_{i, r}$. 
To complete the classification of the irreducible $U(\mathfrak{g})_{\mathbb{F}}$-modules in terms of highest weights, it remains to prove that for every $\lambda \in P^{+}$, there exists an irreducible $U(\mathfrak{g})_{\mathbb{F}}$-module having $\lambda$ as highest weight. We will use reduction modulo $p$.

Let $V$ be a finite-dimensional $U(\mathfrak{g})$-module, and let $L$ be an admissible lattice for $V$. Setting $L_{\mathbb{F}}=L \otimes_{\mathbb{Z}} \mathbb{F}$, we have $L_{\mathbb{F}}$ is a $U(\mathfrak{g})_{\mathbb{F}}$-module and $\operatorname{dim}_{\mathbb{F}}\left(L_{\mathbb{F}}\right)=\operatorname{dim}_{\mathbb{C}}(V)$. The $U(\mathfrak{g})_{\mathbb{F}}$-module $L_{\mathbb{F}}$ is called a reduction modulo $p$ of $V$ (via $L$ ). If $L$ is a minimal admissible lattice for $V=V^{0}(\lambda)$, then $L_{\mathbb{F}}$ is clearly highest-weight with highest weight $\lambda$. Hence by Proposition 2.3, it has a finite-dimensional irreducible quotient. Let $V(\lambda)$ denote this quotient.

The following statement remains true in positive characteristic.

Proposition 2.5. Let $V$ be a finite-dimensional $U(\mathfrak{g})_{\mathbb{F}-\text { module. The generalized }}$ eigenspaces $V_{\mu}$ are in fact eigenspaces and $\operatorname{dim} V_{\mu}=\operatorname{dim} V_{w \mu}$ for all $w \in \mathcal{W}$.

\section{C. Weyl modules and duality.}

Definition 2.6. Given $\lambda \in P^{+}$, let $W(\lambda)$ be the $U(\mathfrak{g})_{\mathbb{E}}$-module generated by a vector $v$ satisfying

$$
\left(x_{\alpha}^{+}\right)^{\left(p^{k}\right)} v=0, \quad\left(\begin{array}{c}
h_{i} \\
p^{k}
\end{array}\right) v=\left(\begin{array}{c}
\lambda\left(h_{i}\right) \\
p^{k}
\end{array}\right) v, \quad\left(x_{\alpha}^{-}\right)^{(l)} v=0,
$$

for all $\alpha \in R^{+}, i \in I$, and $k, l \in \mathbb{Z}_{+}$with $l>\lambda\left(h_{\alpha}\right)$.

The modules $W(\lambda)$ are called Weyl modules. One can show that every finite-

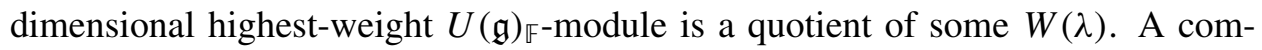
parison between the definition of $W(\lambda)$ and Theorem 2.1(c) hints at the following theorem, which is a consequence of Kempf's vanishing theorem and shows $W(\lambda)$ is universal in the family of finite-dimensional highest-weight modules with highest weight $\lambda$.

Theorem 2.7. Let $\lambda \in P^{+}$, and let $L$ be a minimal admissible lattice for $V^{0}(\lambda)$. Then $W(\lambda)$ is isomorphic to $L_{\mathbb{F}}$. In particular, $W(\lambda)$ is finite dimensional.

We define the notions of lowest-weight vector and lowest-weight module similarly to the corresponding highest-weight notions. It is well known that $V^{0}(\lambda)$ is a lowest-weight module with lowest weight $w_{0} \lambda$, where $w_{0}$ is the longest element of $\mathcal{W}$. Given a highest-weight vector $v$ for $V^{0}(\lambda)$ and a reduced expression $w_{0}=s_{i_{l}} \cdots s_{i_{1}}$, set $m_{i_{k}} \in \mathbb{Z}_{+}$for $k=1, \ldots, l$ to be $\left(s_{i_{k-1}} \cdots s_{i_{1}} \lambda\right)\left(h_{i_{k}}\right)$. Then a lowest-weight vector of $V^{0}(\lambda)$ is given by $v^{\prime}=\left(x_{i_{l}}^{-}\right)^{\left(m_{i_{l}}\right)} \cdots\left(x_{i_{1}}^{-}\right)^{\left(m_{i_{1}}\right)} v$, and moreover $v=\left(x_{i_{1}}^{+}\right)^{\left(m_{i_{1}}\right)} \cdots\left(x_{i_{l}}^{+}\right)^{\left(m_{i_{l}}\right)} v^{\prime}$. This shows that the image of $v^{\prime}$ in the irreducible quotient of $W(\lambda)$ is nonzero and:

Corollary 2.8. For all $\lambda \in P^{+}, W(\lambda)$ and $V(\lambda)$ are lowest-weight modules with lowest weight $w_{0} \lambda$. 
Since $U(\mathfrak{g})_{\mathbb{F}}$ is a Hopf algebra, given a $U(\mathfrak{g})_{\mathbb{F}}$-module $V$, one can equip the dual vector space $V^{*}$ with the structure of a $U(\mathfrak{g})_{\mathbb{F}}$-module, where the action of $x \in U(\mathfrak{g})_{\mathbb{F}}$ on $f \in V^{*}$ is given by $(x f)(v)=f(S(x) v)$ for all $v \in V$.

Proposition 2.9. Let $V$ be a finite-dimensional $U(\mathfrak{g})_{\mathbb{F}-\text { module. Then }}$

(a) The natural isomorphism of vector spaces $V \rightarrow V^{* *}$ is a $U(\mathfrak{g})_{\mathbb{F}}$-module isomorphism.

(b) If $V=V(\lambda)$ for $\lambda \in P^{+}$, then $V^{*} \cong V\left(-w_{0} \lambda\right)$.

Proof. Part (a) is immediate because $S^{2}$ is the identity. Now if $V$ is irreducible, it follows from (a), since duality preserves exact sequences, that $V^{*}$ is also irreducible. From (1-6) we conclude that $V_{\mu} \neq 0$ if and only if $V_{-\mu}^{*} \neq 0$. The final claim now follows immediately from Corollary 2.8 .

2D. Tensor products. We now recall Steinberg's tensor product theorem [Steinberg 1963]. We sketch only the part of the proof which will be relevant for Section 3. Our argument essentially follows the one given in [Cline et al. 1980]. We shall use the following lemma and refer to these references for its proof. Let $P_{p}^{+}=\left\{\lambda \in P^{+}: \lambda\left(h_{i}\right)<p\right.$, for all $\left.i \in I\right\}$.

Lemma 2.10. Let $\lambda, \mu \in P_{p}^{+}-\{0\}$. Then $V(\lambda)$ is irreducible as a $\mathfrak{g}_{\mathbb{F}}$-module and $V(\lambda) \otimes V(\mu)$ is reducible as a $U(\mathfrak{g})_{\mathbb{F}}$-module.

Theorem 2.11. For $\lambda \in P^{+}$, let $\lambda_{k}$ be the unique elements of $P_{p}^{+}$such that $\lambda=$ $\sum_{k=0}^{m} p^{k} \lambda_{k}$. Then $V(\lambda) \cong \otimes_{k} V\left(p^{k} \lambda_{k}\right)$. Moreover, if $\mu_{j} \in P_{p}^{+}-\{0\}$ and $l_{j} \in \mathbb{Z}_{+}$ for $j=0, \ldots, n$ are such that $\otimes_{j=0}^{n} V\left(p^{l_{j}} \mu_{j}\right) \cong V(\lambda)$, then $m=n$ and (up to reordering) $\mu_{k}=\lambda_{k}$ and $l_{k}=k$ for all $k$.

Proof. For any $\mu \in P_{p}^{+}$and $k \in \mathbb{Z}_{+}$we have $V\left(p^{k} \mu\right) \cong V(\mu)^{\phi^{k}}$ (see Section 1D). Therefore $\left(x_{\alpha}^{ \pm}\right)^{\left(p^{l}\right)} V\left(p^{k} \mu\right)=0$ if $l<k$. Let $v_{k}$ be highest-weight vectors for $V\left(p^{k} \lambda_{k}\right)$, let $V^{\prime}=\otimes_{k=1}^{m} V\left(p^{k} \lambda_{k}\right)$, and let $v=\sum_{i} w_{i} \otimes w_{i}^{\prime} \in V\left(\lambda_{0}\right) \otimes V^{\prime}$, where $w_{i}^{\prime}$ are linearly independent. Then $x_{\alpha}^{+} v=\sum_{i}\left(x_{\alpha}^{+} w_{i}\right) \otimes w_{i}^{\prime}$. Since $V\left(\lambda_{0}\right)$ is irreducible as $\mathfrak{g}_{\mathbb{F}}$-module, it follows that $x_{\alpha}^{+} v=0$ only if $v=v_{0} \otimes v^{\prime}$ for some $v^{\prime} \in V^{\prime}$. Now let $V^{\prime \prime}=\bigotimes_{k=2}^{m} V\left(p^{k} \lambda_{k}\right)$ and $v=v_{0} \otimes\left(\sum_{i} w_{i}^{\prime} \otimes w_{i}^{\prime \prime}\right) \in V\left(\lambda_{0}\right) \otimes V\left(p \lambda_{1}\right) \otimes V^{\prime \prime}$, where the $w_{i}^{\prime \prime}$ are linearly independent. Then $\left(x_{\alpha}^{+}\right)^{(p)} v=v_{0} \otimes\left(\sum_{i}\left(\left(x_{\alpha}^{+}\right)^{(p)} w_{i}^{\prime}\right) \otimes w_{i}^{\prime \prime}\right)$. Since $V\left(\lambda_{1}\right)$ is irreducible as $\mathfrak{g}_{\mathbb{F}}$-module, it follows that $\left(x_{\alpha}^{+}\right)^{(p)} v=0$ only if $v=$ $v_{0} \otimes v_{1} \otimes v^{\prime \prime}$ for some $v^{\prime \prime} \in V^{\prime \prime}$. Continuing like this we see that $\bigotimes_{k=0}^{m} V\left(p^{k} \lambda_{k}\right)$ is irreducible. Since it is clearly a highest-weight module with highest-weight $\lambda$, the first statement is proved. On the other hand, we must have $\lambda_{k}=\sum_{j \in J_{k}} \mu_{j}$, where $J_{k}=\left\{j: l_{j}=k\right\}$. Therefore, if $\left\{\mu_{j}\right\}$ were not as stated, there would clearly exist $j \neq j^{\prime}$ such that $l_{j}=l_{j^{\prime}}$. The lemma above would then imply $V\left(p^{l_{j}} \mu_{j}\right) \otimes V\left(p^{l_{j^{\prime}}} \mu_{j^{\prime}}\right)$ is reducible and hence also $\bigotimes_{j=0}^{n} V\left(p^{l_{j}} \mu_{j}\right)$. 
Remark. One reason Theorem 2.11 is important comes from the fact that the (finitely many) modules $V(\lambda)$ for $\lambda \in P_{p}^{+}$are irreducible as modules for the subalgebra of $U(\mathfrak{g})_{\mathbb{F}}$ generated by $x_{\alpha}^{ \pm}$(since they are irreducible as $\mathfrak{g}_{\mathbb{F}}$-modules). This is a finite-dimensional algebra, called the restricted universal enveloping algebra of $\mathfrak{g}_{\mathbb{F}}$. Hence, the study of finite-dimensional irreducible $U(\mathfrak{g})_{\mathbb{F}}$-modules is reduced to the study of finitely many modules for a finite-dimensional algebra.

\section{Finite-dimensional $U(\tilde{\mathfrak{g}})_{\mathbb{F}}$-modules}

We next establish some basic results of the category of finite-dimensional $U(\tilde{\mathfrak{g}})_{\mathbb{F}}-$ modules, such as the classification of the irreducible ones and the characterization of the universal highest-weight modules.

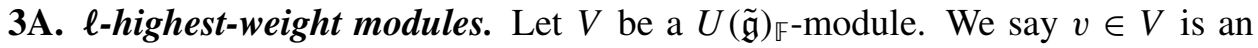
$\ell$-weight vector if it is an eigenvector for the action of $U(\tilde{\mathfrak{h}})_{\mathbb{F}}$, that is, if there exist $z_{i, k}, \varpi_{i, r} \in \mathbb{F}$ such that

$$
\left(\begin{array}{c}
h_{i} \\
p^{k}
\end{array}\right) v=z_{i, k} v \quad \text { and } \quad \Lambda_{i, r} v=\varpi_{i, r} v
$$

for all $i \in I$ and all $r, k \in \mathbb{Z}, k \geq 0$. The corresponding functional $\varpi \in\left(U(\tilde{\mathfrak{h}})_{\mathbb{F}}\right)^{*}$ is called the $\ell$-weight of $v$. If $v$ is an $\ell$-weight vector and $\left(x_{\alpha, r}^{+}\right)^{(k)} v=0$ for all $\alpha \in R^{+}$and all $r, k \in \mathbb{Z}$ with $k>0$, we say $v$ is an $\ell$-highest-weight vector. If $V$ is generated by an $\ell$-highest-weight vector, we say $V$ is an $\ell$-highest-weight module.

Given a finite-dimensional $U(\tilde{\mathfrak{g}})_{\mathbb{F}}$-module $V$ we know from Section 2 that $V$ can be written as the direct sum of its weight spaces when regarded as $U(\mathfrak{g})_{\mathbb{F}}$-module:

$$
V=\bigoplus_{\mu \in P} V_{\mu}
$$

Since $U(\tilde{\mathfrak{h}})_{\mathbb{F}}$ is a commutative algebra, we can also write the decomposition of $V$ into direct sum of generalized eigenspaces for the action of $U(\tilde{\mathfrak{h}})_{\mathbb{F}}$ :

$$
V=\bigoplus_{\varpi \in\left(U(\tilde{\mathfrak{h}})_{\mathrm{F}}\right)^{*}} V_{\varpi}
$$

We next establish a set of relations satisfied by all finite-dimensional $\ell$-highestweight modules.

Proposition 3.1. Let $V$ be a finite-dimensional $U(\tilde{\mathfrak{g}})_{\mathbb{F}}$-module, $\lambda \in P^{+}$, and $v \in V_{\lambda}$ be such that

$$
\left(x_{\alpha, s}^{+}\right)^{(k)} v=0 \quad \text { and } \quad \Lambda_{i, s} v=\omega_{i, s} v,
$$

for all $\alpha \in R^{+}, i \in I, k, s \in \mathbb{Z}$ with $k>0$, and some $\omega_{i, s} \in \mathbb{F}$. Then

$$
\left(x_{\alpha, s}^{-}\right)^{(k)} v=\Lambda_{i, \pm r} v=0 \quad \text { for all } k>\lambda\left(h_{\alpha}\right), r>\lambda\left(h_{i}\right), \text { and } s \in \mathbb{Z} \text {. }
$$


Moreover, $\omega_{i, \pm \lambda\left(h_{i}\right)} \neq 0$ and there exist polynomials $f_{i} \in \mathbb{F}\left[t_{0}, t_{1}, \ldots, t_{\lambda\left(h_{i}\right)}\right]$, depending only on $\lambda\left(h_{i}\right)$, such that

$$
\omega_{i,-r}=f_{i}\left(\omega_{i, \lambda\left(h_{i}\right)}^{-1}, \omega_{i, 1}, \ldots, \omega_{i, \lambda\left(h_{i}\right)}\right) \quad \text { for all } r=1, \ldots, \lambda\left(h_{i}\right) .
$$

Proof. For each $r \in \mathbb{Z}$ and $\alpha \in R^{+}$, the elements $\left(x_{\alpha, \pm r}^{ \pm}\right)^{(k)}$ for $k \in \mathbb{Z}_{+}$generate a subalgebra $U\left(\tilde{\mathfrak{g}}_{\alpha, r}\right)_{\mathbb{F}}$ of $U(\tilde{\mathfrak{g}})_{\mathbb{F}}$ isomorphic to $U\left(\mathfrak{s l}_{2}\right)_{\mathbb{F}}$. Hence, the equality $\left(x_{\alpha, r}^{-}\right)^{(k)} v=0$ for $k>\lambda\left(h_{\alpha}\right)$ follows because $v$ generates a (finite-dimensional) highest-weight module for this subalgebra, which is then isomorphic to a quotient of the Weyl module $W\left(\lambda\left(h_{\alpha}\right)\right)$.

Setting $\alpha=\alpha_{i}, s=0$, and $l=k=r$ in (1-11), we get $\Lambda_{i, \pm r} v=0$ for $r>\left|\lambda\left(h_{i}\right)\right|$. Now, choosing $r=\lambda\left(h_{i}\right)$, we see that $\omega_{i, \pm \lambda\left(h_{i}\right)} \neq 0$. In fact, since $W=U(\tilde{\mathfrak{g}})_{\mathbb{F}} v$ is a finite-dimensional representation for $U(\mathfrak{g})_{\mathbb{F}}$ having $W_{\lambda}=\mathbb{F} v$ as its highestweight space by Equation (1-9), it follows that $\lambda-(r+m) \alpha_{i}$ is not a weight of $W$ for any $m>0$. Therefore $\left(x_{i}^{-}\right)^{(m)}\left(x_{i, \pm 1}^{-}\right)^{(r)} v=0$ for all $m \in \mathbb{N}$. On the other hand, by considering the subalgebra $U\left(\tilde{\mathfrak{g}}_{\alpha_{i}, \mp 1}\right)_{\mathbb{F}}$, we see that $\left(x_{i, \pm 1}^{-}\right)^{(r)} v \neq 0$. It follows that $\left(x_{i, \pm 1}^{-}\right)^{(r)} v$ generates a lowest-weight finite-dimensional representation of $U\left(\tilde{\mathfrak{g}}_{\alpha_{i}, 0}\right)_{\mathbb{F}}$, and in particular $0 \neq\left(x_{i}^{+}\right)^{(r)}\left(x_{i, \pm 1}^{-}\right)^{(r)} v=\Lambda_{i, \pm r} v$.

For the last statement, we proceed by induction on $r=1, \ldots, \lambda\left(h_{i}\right)=m_{i}$. Setting $\alpha=\alpha_{i}, s=0, l=m_{i}$, and $k=l+r$ in (1-11), we get

$$
\omega_{i, m_{i}}\left(x_{i, 1}^{-}\right)^{(r)} v+\sum_{j=1}^{m_{i}} \omega_{i, m_{i}-j} Y_{j} v=0
$$

where $\omega_{i, 0}=1$ and $Y_{j}$ is the sum of the monomials $\left(x_{i, 1}^{-}\right)^{\left(k_{1}\right)} \cdots\left(x_{i, m_{i}+1}^{-}\right)^{\left(k_{m_{i}+1}\right)}$ such that $\sum_{n} k_{n}=r$ and $\sum_{n} n k_{n}=r+j$. Now, since $-r<r+j-2 r<m_{i}$, it is not difficult to see that $\left(x_{i,-2}^{+}\right)^{(r)} Y_{j} \in U(\tilde{\mathfrak{g}})_{\mathbb{F}} U\left(\tilde{\mathfrak{n}}^{+}\right)_{\mathbb{F}}^{0}+H_{j}$, where $H_{j}$ is a linear combination of monomials of the form $\Lambda_{i, r_{1}} \cdots \Lambda_{i, r_{m}}$ such that $-r<r_{j}<m_{i}$. Moreover, $\left(x_{i,-2}^{+}\right)^{(r)}\left(x_{i, 1}^{-}\right)^{(r)} \in(-1)^{r} \Lambda_{i,-r}+U(\tilde{\mathfrak{g}})_{\mathbb{F}} U\left(\tilde{\mathfrak{n}}^{+}\right)_{\mathbb{F}}^{0}$ by (1-11). Hence,

$$
\begin{aligned}
0 & =\left(x_{i,-2}^{+}\right)^{(r)}\left(\omega_{i, m_{i}}\left(x_{i, 1}^{-}\right)^{(r)} v+\sum_{j=1}^{m_{i}} \omega_{i, m_{i}-j} Y_{j} v\right) \\
& =(-1)^{s} \omega_{i,-r} \omega_{i, m_{i}} v+\sum_{j=1}^{m_{i}} \omega_{i, m_{i}-j} H_{j} v .
\end{aligned}
$$

From here it is easy to deduce the last statement.

We would like to be more precise about the last statement of the previous proposition; compare [Chari and Pressley 2001, Proposition 1.1(v)]. Namely, we want to prove that

$$
\Lambda_{i, \lambda\left(h_{i}\right)} \Lambda_{i,-r} v=\Lambda_{i, \lambda\left(h_{i}\right)-r} v \quad \text { for all } i \in I, 0 \leq r \leq \lambda\left(h_{i}\right)
$$


In other words, given $v, \lambda$, and $\omega_{i, r}$ as in the proposition and setting

$$
\boldsymbol{\omega}_{i}(u)=1+\sum_{r=1}^{\lambda\left(h_{i}\right)} \omega_{i, r} u^{r}
$$

we want to show that $\Lambda_{i}^{-}(u) v=\omega_{i}^{-}(u) v$, where for a polynomial $f(u)=\prod_{j}(1-$ $\left.a_{j} u\right) \in \mathbb{F}[u]$, we set $f^{-}(u)=\prod_{j}\left(1-a_{j}^{-1} u\right.$ ) (when convenient we shall also write $\left.f=f^{+}\right)$. The element $\left(\omega_{i}\right)_{i \in I}$ is called the Drinfeld polynomial of the $\ell$-highestweight module generated by $v$. We denote by $\mathscr{P}_{\mathbb{F}}^{+}$the multiplicative monoid consisting of all $|I|$-tuples of the form $\omega=\left(\omega_{i}\right)_{i \in I}$ where each $\omega_{i}$ is a polynomial in $\mathbb{E}[u]$ with constant term 1 . The differential equations techniques used in [Chari and Pressley 2001] for proving (3-12) do not work in positive characteristic. However, in light of Proposition 3.1, it suffices to exhibit for each $\omega \in \mathscr{P}_{\mathbb{F}}^{+}$one finitedimensional $\ell$-highest-weight module with $\ell$-highest weight $\omega$ on which (3-12) is satisfied. This will be done in the next subsection.

We end this subsection introducing additional notation. The multiplicative group corresponding to $\mathscr{P}_{\mathbb{F}}^{+}$will be denoted by $\mathscr{P}_{\mathbb{F}}$. We let wt $: \mathscr{P}_{\mathbb{F}} \rightarrow P$ be the unique group homomorphism such that $\operatorname{wt}(\boldsymbol{\omega})=\sum_{i \in I} \operatorname{deg}\left(\boldsymbol{\omega}_{i}\right) \omega_{i}$ for all $\boldsymbol{\omega} \in \mathscr{P}_{\mathbb{F}}^{+}$. We also have an injective group homomorphism $\mathscr{P}_{\mathbb{F}} \rightarrow\left(U(\tilde{\mathfrak{h}})_{\mathbb{F}}\right)^{*}$ given as follows. Any element $\varpi \in \mathscr{P}_{\mathbb{F}}$ can be written uniquely as $\boldsymbol{\omega} \boldsymbol{\pi}^{-1}$, where $\boldsymbol{\omega}, \boldsymbol{\pi} \in \mathscr{P}_{\mathbb{F}}^{+}$are such that $\boldsymbol{\omega}_{i}, \boldsymbol{\pi}_{i}$ are coprime for all $i \in I$. Its image $\bar{\varpi} \in\left(U(\tilde{\mathfrak{h}})_{\mathbb{F}}\right)^{*}$ is defined by

$$
\bar{\varpi}\left(\left(\begin{array}{c}
h_{i} \\
p^{k}
\end{array}\right)\right)=\left(\begin{array}{c}
\mathrm{wt}(\varpi)\left(h_{i}\right) \\
p^{k}
\end{array}\right) \quad \text { and } \quad \bar{\varpi}\left(\Lambda_{i}^{ \pm}(u)\right)=\varpi_{i}^{ \pm}(u)
$$

for all $k \in \mathbb{Z}_{+}$, and where $\varpi_{i}^{+}=\varpi_{i}$ and $\varpi_{i}^{-}=\omega_{i}^{-}\left(\pi_{i}^{-}\right)^{-1}$. The second equality is that of power series in $u$, obtained by expanding $\left(\pi_{i}^{ \pm}\right)^{-1}$ as a product of geometric power series. We shall identify $\mathscr{P}_{\mathbb{F}}$ with its image in $\left(U(\tilde{\mathfrak{h}})_{\mathbb{F}}\right)^{*}$ and refer to its elements as the integral $\ell$-weights. Similarly, we call the elements in $\mathscr{P}_{\mathbb{F}}^{+}$the dominant integral $\ell$-weights.

3B. Classification of irreducible modules. If $V$ is a finite-dimensional irreducible $U(\tilde{\mathfrak{g}})_{\mathbb{F}}$-module, then, proceeding as in the proof of Theorem 2.4 , we see that $V$ is generated by a vector $v$ satisfying

$$
\left(x_{\alpha, r}^{+}\right)^{\left(p^{k}\right)} v=0, \quad\left(\begin{array}{c}
h_{i} \\
p^{k}
\end{array}\right) v=\left(\begin{array}{c}
\lambda\left(h_{i}\right) \\
p^{k}
\end{array}\right) v, \quad \Lambda_{i, r} v=\omega_{i, r} v
$$

for all $\alpha \in R^{+}, i \in I, r, k \in \mathbb{Z}$ with $k \geq 0$, and some $\lambda \in P^{+}$and $\omega_{i, r} \in \mathbb{F}$. In particular, we have an immediate corollary of Proposition 3.1:

Corollary 3.2. Every finite-dimensional irreducible $U(\tilde{\mathfrak{g}})_{\mathbb{F}}$-module is an $\ell$-highestweight module whose $\ell$-highest weight lies in $\mathscr{P}_{\mathbb{F}}^{+}$. 
We now introduce an important class of $U(\tilde{\mathfrak{g}})_{\mathbb{F}}$-modules, called evaluation representations.

Proposition 3.3. For $a \in \mathbb{F}^{\times}$, there exists a surjective algebra homomorphism $\operatorname{hev}_{a}: U(\tilde{\mathfrak{g}})_{\mathbb{F}} \rightarrow U(\mathfrak{g})_{\mathbb{F}}$ mapping $\left(x_{\alpha, r}^{ \pm}\right)^{(k)}$ to $a^{r k}\left(x_{\alpha}^{ \pm}\right)^{(k)}$. In particular, $\operatorname{hev}_{a}\left(\Lambda_{\alpha, r}\right)=$ $(-a)^{r}\left(\begin{array}{c}h_{\alpha} \\ |r|\end{array}\right)$.

We call hev ${ }_{a}$ the hyperevaluation map at $a$.

Proof. First observe that the formal evaluation map ev on $U(\tilde{\mathfrak{g}})$ (see Lemma 1.1) sends $U(\tilde{\mathfrak{g}})_{\mathbb{Z}}$ to $U(\mathfrak{g})_{\mathbb{Z}} \otimes \mathbb{Z}\left[t, t^{-1}\right]$. Hence, by reducing ev modulo $p$, we obtain the formal hyperevaluation map hev $: U(\tilde{\mathfrak{g}})_{\mathbb{F}} \rightarrow U(\mathfrak{g})_{\mathbb{F}} \otimes \mathbb{F}\left[t, t^{-1}\right]$. The statements of the proposition are now obvious (compare the definition of $\mathrm{ev}_{a}$ and (1-3)).

Given any $U(\mathfrak{g})_{\mathbb{F}}$-module $V$, let $V(a)$ be the pullback of $V$ by hev $a . V(a)$ is called the evaluation representation with spectral parameter $a$ corresponding to $V$. For $a \in \mathbb{F}^{\times}$and $\mu \in P$, let $\omega_{\mu, a}$ be the element in $\mathscr{P}_{\mathbb{F}}$ whose $i$-th entry is $(1-a u)^{\mu\left(h_{i}\right)}$ for $i \in I$. If $V$ is a $U(\mathfrak{g})_{\mathbb{F}}$-highest-weight module of highest weight $\lambda \in P^{+}$, it is easy to see that $V(a)$ is an $\ell$-highest-weight module with Drinfeld polynomial $\boldsymbol{\omega}_{\lambda, a} \in \mathscr{P}_{\mathbb{F}}^{+}$and that the action of $\Lambda_{i}^{-}(u)$ on the $\ell$-highest vector is given by (3-12). We shall denote the evaluation representation by $V(\lambda, a)$ when $V=V(\lambda)$ and by $W(\lambda, a)$ when $V=W(\lambda)$.

If $\lambda \in P_{p}^{+}$, it is easy to see that $V\left(p^{k} \lambda, a\right)$ is isomorphic to $V\left(\lambda, a^{p^{k}}\right)^{\tilde{\phi}^{k}}$, where $\tilde{\phi}$ is the Frobenius homomorphism defined in Section 1D. Moreover, for any $\lambda \in P^{+}$, Theorem 2.11 implies

(3-13) $V(\lambda, a) \cong \bigotimes_{k} V\left(p^{k} \lambda_{k}, a\right)$, where $\lambda_{k} \in P_{p}^{+}$are such that $\lambda=\sum_{k} p^{k} \lambda_{k}$.

We now prove the following version of Steinberg's tensor product theorem for hyper loop algebras.

Theorem 3.4. If $\mu_{j} \in P_{p}^{+}-\{0\}, a_{j} \in \mathbb{F}^{\times}$, and $l_{j} \in \mathbb{Z}_{+}$for $j=0, \ldots, n$, then $V=\bigotimes_{j} V\left(p^{l_{j}} \mu_{j}, a_{j}\right)$ is irreducible if and only if $a_{j} \neq a_{j^{\prime}}$ whenever $l_{j}=l_{j^{\prime}}$.

Proof. The proof is a combination of the arguments used in Theorem 2.11 and [Chari and Pressley 1986, Theorem 1.7]. First consider the case $V=V\left(p^{l} \lambda, a\right) \otimes$ $V\left(p^{l} \mu, b\right)$, where $\lambda, \mu \in P_{p}^{+}$, and let $v=\sum_{j} v_{j} \otimes w_{j} \in V$ be such that $w_{j}$ are linearly independent. Using (1-4) we get

$$
\left(x_{\alpha, r}^{+}\right)^{(k)} v=\sum_{j} \sum_{l+m=k} a^{r l} b^{r m}\left(\left(x_{\alpha}^{+}\right)^{(l)} v_{j}\right) \otimes\left(\left(x_{\alpha}^{+}\right)^{(m)} w_{j}\right) .
$$

Hence, if $a=b$, this implies $\left(x_{\alpha, r}^{+}\right)^{(k)} v=a^{r k}\left(x_{\alpha}^{+}\right)^{(k)} v$, and it follows that, if $v$ generates a $U(\mathfrak{g})_{\mathbb{F}}$-submodule of $V$, it also generates a $U(\tilde{\mathfrak{g}})_{\mathbb{F}}$-submodule of $V$. This proves the "only if" part. 
Conversely, for each $l \in \mathbb{Z}_{+}$, let $J_{l}=\left\{j: l_{j}=l\right\}$ and $V_{l}=\bigotimes_{j \in J_{l}} V\left(p^{l} \mu_{j}, a_{j}\right)$, so that $V \cong \bigotimes_{l: J_{l} \neq \varnothing} V_{l}$. Now observe that

$$
V_{l} \cong\left(\bigotimes_{j \in J_{l}} V\left(\mu_{j}, a_{j}^{p^{l}}\right)\right)^{\tilde{\phi}^{l}} .
$$

The same arguments used in [Chari and Pressley 1986, Theorem 1.7] show that $\bigotimes_{j \in J_{l}} V\left(\mu_{j}, a_{j}^{p^{l}}\right)$ is irreducible as a $\tilde{\mathfrak{g}}_{\mathbb{F}}$-module, and therefore $V_{l}$ is irreducible as an $U(\tilde{\mathfrak{g}})_{\mathbb{F}}$-module. Now let $\left\{l_{1}, \ldots, l_{m}\right\}=\left\{l: J_{l} \neq \varnothing\right\}$ and suppose $l_{1}<l_{2}<\cdots<l_{m}$. Set $V^{\prime}=\bigotimes_{j=2}^{m} V_{l_{j}}$ and let $v=\sum_{i} w_{i} \otimes w_{i}^{\prime} \in V_{l_{1}} \otimes V^{\prime}$ such that $w_{i}^{\prime}$ are linearly independent. Then

$$
\left(x_{\alpha, r}^{+}\right)^{\left(p^{l_{1}}\right)} v=\sum_{i}\left(\left(x_{\alpha, r}^{+}\right)^{\left(p^{l_{1}}\right)} w_{i}\right) \otimes w_{i}^{\prime},
$$

and we see that $v$ is an $\ell$-highest-weight vector only if $v=v_{1} \otimes v^{\prime}$ where $v_{1}$ is an $\ell$-highest-weight vector for $V_{l_{1}}$, since we already know that $V_{l_{j}}$ is irreducible. Proceeding inductively like the proof of Theorem 2.11, we conclude that $v$ must be a multiple of the tensor product of the $\ell$-highest-weight vectors.

As a corollary, we obtain the classification of the irreducible representations for $U(\tilde{\mathfrak{g}})_{\mathbb{F}}$; see [Chari 1986; Chari and Pressley 1986; 2001]. It is easy to see that every element $\varpi \in \mathscr{P}_{\mathbb{F}}$ can be uniquely decomposed as $\varpi=\prod_{j} \omega_{\mu_{j}, a_{j}}$ for some $\mu_{j} \in P$ and $a_{i} \neq a_{j}$.

Corollary 3.5. (a) If $\omega=\prod \omega_{\lambda_{j}, a_{j}} \in \mathscr{P}_{\mathbb{F}}^{+}$with $a_{i} \neq a_{j}$ for $i \neq j$, and $\lambda_{j}=$ $\sum_{k} p^{k} \lambda_{j, k}$ with $\lambda_{j, k} \in P_{p}^{+}$, then $V=\bigotimes_{j, k} V\left(p^{k} \lambda_{j, k}, a_{j}\right)$ is an irreducible $U(\tilde{\mathfrak{g}})_{\mathbb{F}}$-module with $\ell$-highest weight $\boldsymbol{\omega}$. In particular, (3-12) holds for $V$.

(b) The isomorphism classes of irreducible finite-dimensional $U(\tilde{\mathfrak{g}})_{\mathbb{F}}$-modules are in one-to-one correspondence with the elements of $\mathscr{P}_{\mathbb{F}}^{+}$.

Proof. It is immediate from Theorem 3.4 that $V$ is irreducible and therefore has an $\ell$-highest weight in $\mathscr{P}_{\mathbb{F}}^{+}$. To see that this $\ell$-highest weight is $\omega$, one easily computes the action of $\Lambda_{i}^{+}(u)$ on the $\ell$-highest-weight vector using (1-5) and observing that each tensor factor is an evaluation representation. The proof of (3-12) is completed in a similar way by computing the action of $\Lambda_{i}^{-}(u)$ on the $\ell$-highest-weight vector. This completes the proof of part (a), from which part (b) follows immediately.

Given $\omega \in \mathscr{P}_{\mathbb{F}}^{+}$, let us denote by $V(\boldsymbol{\omega})$ an irreducible $U(\tilde{\mathfrak{g}})_{\mathbb{F}}$-module with $\ell$ highest weight $\omega$. If $\omega=\prod \omega_{\lambda_{j}, a_{j}} \in \mathscr{P}_{\mathbb{F}}^{+}$with $a_{i} \neq a_{j}$ for $i \neq j$, it follows from (3-13) and the corollary above that $V(\omega) \cong \bigotimes_{j} V\left(\lambda_{j}, a_{j}\right)$.

Corollary 3.6. If $V$ is a finite-dimensional $U(\tilde{\mathfrak{g}})_{\mathbb{E}}$-module, then $V_{\varpi} \neq 0$ only if $\varpi \in \mathscr{P}_{\mathbb{F}}$ and $V_{\mu}=\bigoplus_{\varpi: \operatorname{wt}(\varpi)=\mu} V_{\varpi}$. 
Proof. It suffices to prove the claim for irreducible representations. Using (1-5) and the last corollary, it suffices to consider the irreducible evaluation representations $V=V(\lambda, a)$ with $\lambda \in P^{+}$. But in this case we have $V_{\mu}=V_{\boldsymbol{\omega}_{\mu, a}}$ (see also Proposition 4.11 below and its corollary).

We end the subsection computing the dual representation of a given irreducible one. Let $V$ be a finite-dimensional $U(\tilde{\mathfrak{g}})_{\mathbb{F}}$-module. Exactly as in the case of $U(\mathfrak{g})_{\mathbb{F}}$, we see that the dual vector space $V^{*}$ can be equipped with a $U(\tilde{\mathfrak{g}})_{\mathbb{F}}$-module structure and that $V^{* *}$ is naturally isomorphic to $V$. If $W$ is another finite-dimensional $U(\tilde{\mathfrak{g}})_{\mathbb{F}}$-module, the usual Hopf algebra arguments prove that we have a natural isomorphism of $U(\tilde{\mathfrak{g}})_{\mathbb{F}}$-modules

$$
(V \otimes W)^{*} \cong W^{*} \otimes V^{*} .
$$

Given $\varpi=\prod_{j} \omega_{\mu_{j}, a_{j}} \in \mathscr{P}_{\mathbb{F}}$ for $a_{i} \neq a_{j}$, set $\varpi^{*}=\prod_{j} \boldsymbol{\omega}_{-w_{0} \mu_{j}, a_{j}}$. We have:

Proposition 3.7. Let $\omega \in \mathscr{P}_{\mathbb{F}}^{+}$and $V=V(\omega)$. Then $V^{*} \cong V\left(\omega^{*}\right)$.

Proof. Due to Theorem 3.4 and (3-14), it suffices to consider the case $\omega=\omega_{\lambda, a}$ for some $a \in \mathbb{F}^{\times}$and $\lambda \in P^{+}$. Since in this case $V$ is an evaluation representation, every weight vector of $V$ is also an $\ell$-weight vector and $V_{\mu}=V_{\boldsymbol{\omega}_{\mu, a}}$. Choose a basis for $V$ consisting of weight vectors. Then it is easy to see using (1-6) and (1-3) that if $v$ is a basis element of weight $\mu$, then its dual vector $v^{*}$ is an $\ell$-weight vector of $\ell$-weight $\omega_{w_{0} \mu, a}$. In particular, since $V^{*} \cong V\left(-w_{0} \lambda\right)$ as $U(\mathfrak{g})_{\mathbb{F}}$, we conclude that $V^{*}$ is the evaluation representation $V\left(-w_{0} \lambda, a\right)$.

3C. The Weyl modules. We now study the universal finite-dimensional $\ell$-highestweight $U(\tilde{\mathfrak{g}})_{\mathbb{F}}$-modules motivated by [Chari and Pressley 2001].

Definition 3.8. Given $\omega=\left(\omega_{i}\right)_{i \in I} \in \mathscr{P}_{\mathbb{F}}^{+}$, let $W(\boldsymbol{\omega})$ be the $U(\tilde{\mathfrak{g}})_{\mathbb{F}}$-module generated by a vector $v$ satisfying

$$
\begin{gathered}
\left(x_{\alpha, r}^{+}\right)^{\left(p^{k}\right)} v=0, \quad\left(\begin{array}{c}
h_{i} \\
p^{k}
\end{array}\right) v=\left(\begin{array}{c}
\operatorname{wt}(\omega)\left(h_{i}\right) \\
p^{k}
\end{array}\right) v, \quad \Lambda_{i, \pm s} v=\left(\omega_{i}^{ \pm}(u)\right)_{s} v, \\
\left(x_{\alpha, r}^{-}\right)^{(l)} v=0,
\end{gathered}
$$

for all $\alpha \in R^{+}, i \in I$, and $k, l, r, s \in \mathbb{Z}$ with $s, k \geq 0$ and $l>\operatorname{wt}(\omega)\left(h_{\alpha}\right)$. Here as before, $\left(\omega_{i}^{ \pm}(u)\right)_{s}$ means the coefficient of $u^{s}$. We call $W(\omega)$ the Weyl module with $\ell$-highest weight $\omega$.

It follows from (1-9) that

$$
W(\boldsymbol{\omega})=\bigoplus_{\mu \leq \mathrm{wt}(\boldsymbol{\omega})} W(\boldsymbol{\omega})_{\mu}
$$

Standard arguments show: 
Proposition 3.9. $W(\omega)$ has a unique maximal submodule and hence a unique irreducible quotient.

In particular, $V(\boldsymbol{\omega})$ is the irreducible quotient of $W(\boldsymbol{\omega})$ for $\boldsymbol{\omega} \in \mathscr{P}_{\mathbb{F}}^{+}$. Moreover, it follows from Proposition 3.1 that every finite-dimensional $\ell$-highest weight module of $\ell$-highest weight $\omega$ is isomorphic to a quotient of $W(\omega)$. Hence, to complete the proof of the universality of $W(\omega)$, it remains to show that it is finite dimensional. We begin with:

Proposition 3.10. If $W(\boldsymbol{\omega})_{\mu} \neq 0$, then $W(\boldsymbol{\omega})_{w \mu} \neq 0$ for all $w \in \mathcal{W}$. In particular, $W(\boldsymbol{\omega})_{\mu} \neq 0$ only if $w_{0} \mathrm{wt}(\boldsymbol{\omega}) \leq \mu \leq \operatorname{wt}(\boldsymbol{\omega})$.

Proof. Using an argument identical to the one used in characteristic zero, it follows from (3-16) that every vector $w \in W(\boldsymbol{\omega})$ lies inside a finite-dimensional $U(\mathfrak{g})_{\mathbb{F}^{-}}$ submodule of $W(\omega)$. Now all the claims follow from the corresponding results for finite-dimensional $U(\mathfrak{g})_{\mathbb{F}}$-modules.

Theorem 3.11. $W(\omega)$ is finite-dimensional for all $\omega \in \mathscr{P}_{\mathbb{F}}^{+}$.

This was proved in [Chari and Pressley 2001] for characteristic zero and for quantum groups, the latter under the assumption that $\mathfrak{g}$ is simply laced; for nonsimply laced it follows from [Beck and Nakajima 2004].

Proof. Set $\lambda=\operatorname{wt}(\omega)$, and let $v$ be an $\ell$-highest-weight vector of $W(\boldsymbol{\omega})$. It suffices to prove that $W(\omega)$ is spanned by the elements

$$
\left(x_{\beta_{1}, s_{1}}^{-}\right)^{\left(k_{1}\right)} \cdots\left(x_{\beta_{m}, s_{m}}^{-}\right)^{\left(k_{m}\right)} v,
$$

with $m, s_{j}, k_{j} \in \mathbb{Z}_{+}$and $\beta_{j} \in R^{+}$such that $s_{j}<\lambda\left(h_{\beta_{j}}\right)$ and $\sum_{j} k_{j} \beta_{j} \leq \lambda-w_{0} \lambda$. The last condition is immediate from the previous proposition. Moreover, the elements $\left(x_{\beta_{1}, s_{1}}^{-}\right)^{\left(k_{1}\right)} \cdots\left(x_{\beta_{m}, s_{m}}^{-}\right)^{\left(k_{m}\right)} v$ with no restriction on $s_{j}$ clearly span $W(\boldsymbol{\omega})$.

Let $\mathscr{R}=R^{+} \times \mathbb{Z} \times \mathbb{Z}_{+}$, and let $\Xi$ be the set of functions $\xi: \mathbb{N} \rightarrow \mathscr{R}$ given by $j \mapsto \xi_{j}=\left(\beta_{j}, s_{j}, k_{j}\right)$ such that $k_{j}=0$ for all $j$ sufficiently large. Also let $\Xi^{\prime}$ be the subset of $\Xi$ consisting of the elements $\xi$ such that $0 \leq s_{j}<\lambda\left(h_{\beta_{j}}\right)$. Given $\xi \in \Xi$, we associate an element $v_{\xi} \in W(\boldsymbol{\omega})$ as above in the obvious way, that is, if $k_{j}=0$ for $j>m$, then $v_{\xi}=\left(x_{\beta_{1}, s_{1}}^{-}\right)^{\left(k_{1}\right)} \cdots\left(x_{\beta_{m}, s_{m}}^{-}\right)^{\left(k_{m}\right)} v$. Define the degree of $\xi$ to be $d(\xi)=\sum_{j} k_{j}$ and the maximal exponent of $\xi$ to be $e(\xi)=\max \left\{k_{j}\right\}$. Clearly $e(\xi) \leq d(\xi)$ and $d(\xi) \neq 0$ implies $e(\xi) \neq 0$. Since there is nothing to be proved when $d(\xi)=0$, we assume from now on that $d(\xi)>0$. Thus let $\boldsymbol{\Xi}_{d, e}$ be the subset of $\Xi$ consisting of those $\xi$ satisfying $d(\xi)=d$ and $e(\xi)=e$, and set $\Xi_{d}=\bigcup_{1 \leq e \leq d} \Xi_{d, e}$.

We prove by induction on $d$ and subinduction on $e$ that if $\xi \in \Xi_{d, e}$ is such that there exists $j$ with either $s_{j}<0$ or $s_{j} \geq \lambda\left(h_{\beta_{j}}\right)$, then $v_{\xi}$ is in the span of vectors associated to elements in $\Xi^{\prime}$. More precisely, given $0<e \leq d \in \mathbb{N}$, induction 
hypothesis says that this statement is true for every $\xi$ that belongs either to $\boldsymbol{\Xi}_{d, e^{\prime}}$ with $e^{\prime}<e$ or to $\Xi_{d^{\prime}}$ with $d^{\prime}<d$.

Observe that (1-11) implies

$$
\left(\left(X_{\beta ; r,+}^{-}(u)\right)^{(k-l)} \Lambda_{\beta}^{+}(u)\right)_{k} v=0
$$

for all $\beta \in R^{+}$and $k, l, r \in \mathbb{Z}$ with $k>\lambda\left(h_{\beta}\right)$ and $1 \leq l \leq k$. We divide the proof according to whether $e=d$ or $e<d$.

When $e=d$, it follows that $v_{\xi}=\left(x_{\beta, s}^{-}\right)^{(e)} v$ for some $\beta \in R^{+}$and $s \in \mathbb{Z}$. Suppose first that $e=1$, and let $l=\lambda\left(h_{\beta}\right)$ and $k=l+1$ in (3-17) to get

$$
\left(x_{\beta, r+1}^{-} \Lambda_{\beta, l}+x_{\beta, r+2}^{-} \Lambda_{\beta, l-1}+\cdots+x_{\beta, r+l+1}^{-}\right) v=0 .
$$

We consider the cases $s \geq l$ and $s<0$ separately and prove the statement by a further induction on $s$ and $|s|$, respectively. If $s \geq l$, this is easily done by setting $r=s-l-1$ in (3-18). Similarly, after observing that $\Lambda_{\beta, l} v \neq 0$, the case $s<0$ is dealt with by setting $r=s-1$ in (3-18). If $e>1$, let $l=e \lambda\left(h_{\beta}\right)$ and $k=l+e$ in (3-17) to obtain

$$
\sum_{n=0}^{\lambda\left(h_{\beta}\right)}\left(x_{\beta, r+1+n}^{-}\right)^{(e)} \Lambda_{\beta, l-e n} v+\text { other terms }=0,
$$

where the other terms belong to the span of elements $v_{\xi^{\prime}}$ with $\xi^{\prime} \in \Xi_{e, e^{\prime}}$ for $e^{\prime}<e$. As before, we argue by induction on $s$ and $|s|$ by setting $r=s-1-\lambda\left(h_{\beta}\right)$ and $r=s-1$ in (3-19), respectively.

For the case $e<d$, the induction hypothesis gives that $0 \leq s_{j}<\lambda\left(h_{\beta_{j}}\right)$ for $j>1$. An easy application of Lemma 1.5 completes the argument in this case.

\section{Reduction modulo $p$}

4A. Introductory remarks and notation. We now start the theory of reduction modulo $p$ for $U(\tilde{\mathfrak{g}})_{\mathbb{K}}$-modules, where $\mathbb{K}$ is a field of characteristic zero. In the case of $U(\mathfrak{g})_{\mathbb{K}}$, it sufficed to prove the existence of admissible lattices for the irreducible modules because the underlying abelian category was semisimple. The category $\widetilde{\mathscr{C}}_{\mathbb{K}}$ is not semisimple so, even if it is possible to obtain a nice lattice theory for all irreducible modules, one could not guarantee that all of the objects in $\widetilde{\mathscr{C}}_{\mathbb{K}}$ would contain such a lattice. In fact, even for irreducible modules the story is more subtle than the one in the $U(\mathfrak{g})_{\mathbb{K}}$-case since the evaluation maps $\operatorname{hev}_{a}$ do not preserve $\mathbb{Z}$-lattices unless $a= \pm 1$. Still, we will prove that all the $\ell$-highest-weight modules whose coefficients of their Drinfeld polynomials are "good" with respect to $p$ can indeed be reduced modulo $p$. In particular, it will follow that every irreducible $U(\tilde{\mathfrak{g}})_{\mathbb{F}}$-module can be constructed as a quotient of a module obtained by a reduction modulo $p$ process. 
We consider two kinds of lattice theories. The first is a natural generalization of the one reviewed in Theorem 2.2 for $U(\mathfrak{g})$. Namely, in Section 4B, we consider modules containing finitely-generated free $\mathbb{Z}$-submodules that are invariant under the action of $U(\tilde{\mathfrak{g}})_{\mathbb{Z}}$. However, the modules $V^{0}(\lambda, a)$ with $a \in \mathbb{Z}$ for $a \neq \pm 1$ are easily seen not to contain such a lattice. In Section $4 C$, we consider lattices over rings other than $\mathbb{Z}$, namely, over torsion-free discrete valuation rings. We think these lattices are more suitable for studying reduction modulo $p$ in the present context.

If $\mathbb{A}$ is any commutative ring with identity, define $\mathscr{P}_{\mathbb{A}}, \mathscr{P}_{\mathbb{A}}^{+}$in the obvious way (see the definition of $\mathscr{P}_{\mathbb{F}}$ ). Define also $\mathscr{P}_{\mathbb{A}}^{++}$as the subset of $\mathscr{P}_{\mathrm{A}}^{+}$consisting of the elements $\omega$ such that the coefficient of the leading term of $\omega_{i}$ belongs to $A^{\times}$for all $i \in I$. Recall that $\mathbb{A}$ is a discrete valuation ring if it is a local principal ideal domain which is not a field; recall that its residue field is the quotient of $\mathbb{A}$ by its unique maximal ideal. If $\mathbb{A}$ is a discrete valuation ring with residue field $\mathbb{F}, a \in \mathbb{A}$, and $\omega \in \mathscr{P}_{\mathbb{A}}^{+}$, we let $\bar{a}$ and $\bar{\omega}$ be the images of $a$ in $\mathbb{F}$ and of $\omega$ in $\mathscr{P}_{\mathbb{F}}^{+}$, respectively. As before, $\mathbb{F}$ denotes an algebraically closed field of characteristic $p>0$. We shall also denote by $\bar{\omega}$ the image of $\omega \in \mathscr{P}_{\mathbb{Z}}^{+}$in $\mathscr{P}_{\mathbb{F}}^{+}$. We fix a torsion-free discrete valuation $\operatorname{ring} \mathbb{A}$ with residue field $\mathbb{E}$, for instance, the ring of Witt vectors with coefficients in $\mathbb{F}$ [Serre 1979, Section II.5]; we denote by $\mathbb{F}^{0}$ the algebraic closure of the fraction field of A. Given $\boldsymbol{\omega} \in \mathscr{P}_{\mathbb{F}^{0}}^{+}$, we denote by $W^{0}(\boldsymbol{\omega})$ the corresponding $U(\tilde{\mathfrak{g}})_{\mathbb{F}^{0}}$-Weyl module [Chari and Pressley 2001] and by $V^{0}(\omega)$ its irreducible quotient.

\section{B. $\mathbb{Z}$-lattices.}

Definition 4.1. If $V$ is a finite-dimensional $\mathbb{F}^{0}$-vector space, we say that a finitelygenerated free $\mathbb{Z}$-submodule $L$ of $V$ is an ample-lattice for $V$ if $L$ spans $V$ over $\mathbb{F}^{0}$. If the rank of $L$ is equal to the dimension of $V$, then we say $L$ is a lattice for $V$. If $V$ is a $U(\tilde{\mathfrak{g}})_{\mathbb{F}}$-module, we say that an ample-lattice for $V$ is admissible if $L$ is invariant under the action of $U(\tilde{\mathfrak{g}})_{\mathbb{Z}}$.

If $L$ is an admissible ample-lattice for a $U(\tilde{\mathfrak{g}})_{\mathbb{F} 0}$-module $V$, we set $L_{\mathbb{F}}=L \otimes_{\mathbb{Z}} \mathbb{F}$. Thus, $L_{\mathbb{F}}$ is a $U(\tilde{\mathfrak{g}})_{\mathbb{F}}$-module and $\operatorname{rank}(L)=\operatorname{dim}_{\mathbb{F}}\left(L_{\mathbb{F}}\right) \geq \operatorname{dim}_{\mathbb{F} 0}(V)$. It is trivial to see that the modules $V^{0}(\lambda, a)$ with $a \neq \pm 1$ do not contain a finitely-generated $\mathbb{Z}$ submodule invariant under the action of $U(\tilde{\mathfrak{g}})_{\mathbb{Z}}$. In fact, if $v$ is the $\ell$-highest-weight vector, then $\left(\Lambda_{i, \pm \lambda\left(h_{i}\right)}\right)^{k} v=(-a)^{ \pm k \lambda\left(h_{i}\right)} v$ is not a finitely-generated $\mathbb{Z}$-module.

Proposition 4.2. Let $V$ be a finite-dimensional $\ell$-highest weight $U(\tilde{\mathfrak{g}})_{\mathbb{F}^{0}}$-module with $\ell$-highest-weight $\omega \in \mathscr{P}_{\mathbb{Z}}^{++}$and $\ell$-highest-weight vector $v$. Then $L=U(\tilde{\mathfrak{g}})_{\mathbb{Z}} v$ is an admissible ample-lattice for $V$, and $L_{\mathbb{F}}$ is isomorphic to a quotient of $W(\bar{\omega})$. If $V=W^{0}(\omega)$, then $L$ is a lattice.

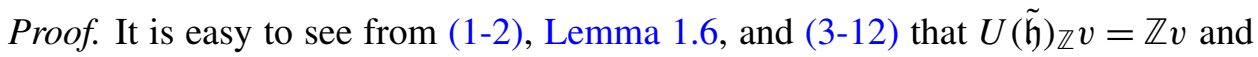
therefore $L=U\left(\tilde{\mathfrak{n}}^{-}\right)_{\mathbb{Z}} v$. Also, $L$ is quite clearly a torsion-free $\mathbb{Z}$-submodule of $V$ 
which is invariant under the action of $U(\tilde{\mathfrak{g}})_{\mathbb{Z}}$. The proof of Theorem 3.11 together with the hypothesis $\omega \in \mathscr{P}_{\mathbb{Z}}^{++}$shows that $L$ is a finitely-generated $\mathbb{Z}$-module which spans $V$ over $\mathbb{F}^{0}$ (the hypothesis $\omega \in \mathscr{P}_{\mathbb{Z}}^{++}$is used to replace the remark $\Lambda_{\beta, l} v \neq 0$ by $\Lambda_{\beta, l} v=a v$ with $a \in \mathbb{Z}^{\times}$). This completes the proof that $L$ is an admissible ample-lattice. Since the image of $v$ in $L_{\mathbb{F}}$ is clearly an $\ell$-highest-weight vector with $\ell$-highest weight $\bar{\omega}$, the second statement follows immediately. The last statement is clear since $L \otimes_{\mathbb{Z}} \mathbb{F}^{0}$ is an $\ell$-highest-weight $U(\tilde{\mathfrak{g}})$-module of $\ell$-highest weight $\omega$ and of dimension at least that of $W^{0}(\boldsymbol{\omega})$, and thus $L \otimes_{\mathbb{Z}} \mathbb{F}^{0} \cong W^{0}(\boldsymbol{\omega})$.

Clearly the only irreducible $U(\tilde{\mathfrak{g}})_{\mathbb{F}}$-modules that can be obtained as a quotient of some $L_{\mathbb{F}}$, where $L$ is as in the proposition, are precisely those whose Drinfeld polynomials $\omega$ lie in $\mathscr{P}_{\mathbb{F}_{p}}^{+}$and whose coefficient in the leading term of $\omega_{i}$ is \pm 1 for all $i \in I$. However, all of the $\ell$-highest-weight $U(\tilde{\mathfrak{g}})_{\mathbb{E}}$-modules $V$ whose Drinfeld polynomial is of the form $\omega_{\lambda, 1}$ can be obtained in this way. In the next section we will see that, for each $a \in \mathbb{F}^{\times}$, there exists an automorphism $\psi_{a}$ of $U(\tilde{\mathfrak{g}})_{\mathbb{F}}$ determined by the assignment $\left(x_{\alpha, r}^{ \pm}\right)^{(k)} \mapsto a^{r k}\left(x_{\alpha, r}^{ \pm}\right)^{(k)}$ for all $\alpha \in R^{+}$and $k, r \in \mathbb{Z}$ with $k>0$. One can then show that the pullback of such $V$ by $\psi_{a}$ is an $\ell$-highestweight module with Drinfeld polynomial $\omega_{\lambda, a}$. Hence, up to twisting by $\psi_{a}$, we obtain all of the evaluation modules $V(\lambda, a)$. The other irreducible modules are then obtained using tensor products.

4C. Lattices over discrete valuation rings. We begin by giving a motivation for considering lattices over discrete valuation rings. Let $\mathbb{P}=\mathbb{Z}_{(p)}$ be the localization of $\mathbb{Z}$ at $\mathbb{Z}-p \mathbb{Z}$, and let $U(\tilde{\mathfrak{g}})_{\mathbb{P}}=U(\tilde{\mathfrak{g}})_{\mathbb{Z}} \otimes_{\mathbb{Z}} \mathbb{P}$. Then $\mathbb{P}$ is a torsion-free discrete valuation ring with residue field $\mathbb{F}_{p}$ and $U(\tilde{\mathfrak{g}})_{\mathbb{P}} \otimes_{\mathbb{P}} \mathbb{F} \cong U(\tilde{\mathfrak{g}})_{\mathbb{F}}$. Let $a \in \mathbb{P}^{\times}, v$ be an $\ell$-highest-weight vector of $V=V^{0}(\lambda, a)$, and $L=U(\tilde{\mathfrak{g}})_{\mathbb{P}} v$. It is easy to see from (1-2), (1-3), and Lemma 1.6 that $U(\tilde{\mathfrak{h}})_{\mathbb{F}} v=\mathbb{P} v$, and therefore $L=U\left(\tilde{\mathfrak{n}}^{-}\right)_{\mathbb{P}} v=$ $\mathbb{P}\left(U\left(\tilde{\mathfrak{n}}^{-}\right)_{\mathbb{Z}} v\right)=\mathbb{P} L^{\prime}$, where $L^{\prime}=U\left(\mathfrak{n}^{-}\right)_{\mathbb{Z}} v$ and $\mathbb{P} L^{\prime}$ is its $\mathbb{P}$-span. Since $L^{\prime}$ is the $\mathbb{Z}$-span of a basis for $V$ by Theorem 2.2, it follows that $L$ is the $\mathbb{P}$-span of the same basis. Thus, setting $L_{\mathbb{F}}=L \otimes_{\mathbb{P}} \mathbb{F}$, we obtain a $U(\tilde{\mathfrak{g}})_{\mathbb{F}}$-module isomorphic to $W(\lambda, \bar{a})$, where $\bar{a}$ is the image of $a$ in $\mathbb{F}_{p}$. In this way we can obtain all evaluation representations of the form $V(\lambda, b)$ for $b \in \mathbb{F}_{p}$ as quotients of the reduction modulo $p$ of the irreducible $U(\tilde{\mathfrak{g}})$-modules $V^{0}(\lambda, a)$, where $a$ is such that $\bar{a}=b$. To obtain $V(\lambda, b)$ for all $b \in \mathbb{F}$, we will have to use in place of $\mathbb{P}$ the bigger discrete valuation ring $\mathbb{A}$ fixed in Section $4 \mathrm{~A}$. Recall that $\mathbb{F}^{0}$ denotes the algebraic closure of the fraction field of A.

Definition 4.3. If $V$ is a finite-dimensional $\mathbb{F}^{0}$-vector space, we say that a finitelygenerated free $\mathbb{A}$-submodule $L$ of $V$ is an $\mathbb{A}$-ample-lattice for $V$ if $L$ spans $V$ over $\mathbb{F}^{0}$. If the rank of $L$ is equal to the dimension of $V$, then we say $L$ is an A-lattice for $V$. If $V$ is a $U(\tilde{\mathfrak{g}})_{\mathbb{F}^{0}}$-module, we say that an ample-lattice for $V$ is admissible if $L$ is invariant under the action of $U(\tilde{\mathfrak{g}})_{\mathbb{A}}=U(\tilde{\mathfrak{g}})_{\mathbb{Z}} \otimes_{\mathbb{Z}} \mathbb{A}$. 
If $L$ is an $\mathbb{A}$-ample-lattice for a $U(\tilde{\mathfrak{g}})_{\mathbb{F} 0}$-module $V$, we set $L_{\mathbb{F}}=L \otimes_{\mathbb{A}} \mathbb{F}$. Then $U(\tilde{\mathfrak{g}})_{\mathbb{F}} \cong U(\tilde{\mathfrak{g}})_{\mathbb{A}} \otimes_{\mathbb{A}} \mathbb{F}$, and $L_{\mathbb{F}}$ is a $U(\tilde{\mathfrak{g}})_{\mathbb{F}}$-module. The next lemma is immediate.

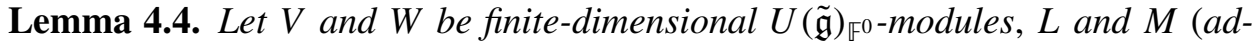
missible) ample-lattices for $V$ and $W$, respectively. Then $L_{\mathbb{A}} M$ is an (admissible) ample-lattice for $V \otimes W$ and $\left(L \otimes_{\mathbb{A}} M\right)_{\mathbb{F}} \cong L_{\mathbb{F}} \otimes M_{\mathbb{E}}$ as $U(\tilde{\mathfrak{g}})_{\mathbb{E}}$-modules.

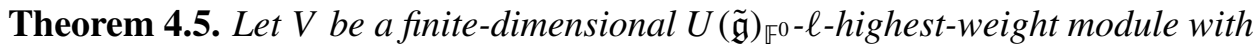
Drinfeld polynomial $\omega \in \mathscr{P}_{\mathbb{A}}^{++}$and $\ell$-highest-weight vector $v$. If $L=U(\tilde{\mathfrak{g}})_{\mathbb{A}} v$ we have

(a) $L$ is an admissible $\mathbb{A}$-ample-lattice for $V$ and $L_{\mathbb{F}}$ is isomorphic to a quotient of $W(\overline{\boldsymbol{\omega}})$;

(b) if $V=W^{0}(\omega)$, then $L$ is a lattice;

(c) if $V=V^{0}(\omega)$ and $\omega=\prod_{j=1}^{m} \omega_{\lambda_{j}, a_{j}}$ with $\lambda_{j} \in P^{+}, a_{j} \in \mathbb{A}^{\times}, a_{i} \neq a_{j}$ when $i \neq j$, then $L$ is a lattice.

Proof. The proofs of parts (a) and (b) are like that of Proposition 4.2 with $\mathbb{A}$ in place of $\mathbb{Z}$.

We now prove (c). When $V$ is an evaluation representation, that is when $m=1$, we proceed as in the motivation at the beginning of $4 \mathrm{C}$ by replacing $\mathbb{P}$ with $\mathbb{A}$, $U(\tilde{\mathfrak{g}})$ with $U(\tilde{\mathfrak{g}})_{\mathbb{F}^{0}}$ and regarding $U(\tilde{\mathfrak{g}})_{\mathbb{Z}}$ as embedded in $U(\tilde{\mathfrak{g}})_{\mathbb{F} 0}$. In the general case we have $V=V^{0}\left(\lambda_{1}, a_{1}\right) \otimes \cdots \otimes V^{0}\left(\lambda_{m}, a_{m}\right)$. Let $v_{j}$ be an $\ell$-highest-weight vector of $V^{0}\left(\lambda_{j}, a_{j}\right)$ so that $v=v_{1} \otimes \cdots \otimes v_{m}$ and set $L^{\prime}=L_{1} \otimes_{\mathbb{A}} \cdots \otimes_{\mathbb{A}} L_{m}$, where $L_{j}=U(\tilde{\mathfrak{g}})_{\AA} v_{j}$. Since each $L_{j}$ is an admissible lattice for $V^{0}\left(\lambda_{j}, a_{j}\right), L^{\prime}$ is an admissible lattice for $V$ by Lemma 4.4. It is clear from (1-4) that $L$ is an $\mathbb{A}$-submodule of $L^{\prime}$. Moreover, by part (a), $L$ is a finitely-generated free $\mathbb{A}$-module which spans $V$, and hence $L=L^{\prime}$, since $\mathbb{A}$ is a principal ideal domain.

We have finished the task of constructing all the irreducible $U(\tilde{\mathfrak{g}})_{\mathbb{F}}$-modules directly as quotients of some $U(\tilde{\mathfrak{g}})_{\mathbb{F}^{0}}$-modules by a reduction modulo $p$ process:

Corollary 4.6. For every $\varpi \in \mathscr{P}_{\mathbb{F}}^{+}$there exists $\omega \in \mathscr{P}_{\mathbb{A}}^{++}$such that $\bar{\omega}=\varpi$ and $V(\varpi)$ is isomorphic to a quotient of $L_{\mathbb{F}}$, where $L=U(\tilde{\mathfrak{g}})_{\mathbb{A}} v$ and $v$ is an $\ell$-highest-weight vector for $W^{0}(\omega)$.

Proof. Write $\varpi=\prod_{j} \omega_{\lambda, b_{j}}$ for $b_{j} \in \mathbb{F}^{\times}$and $b_{i} \neq b_{j}$ with $i \neq j$, and let $a_{j} \in \mathbb{A}^{\times}$ be lifts of $b_{j}$ to $\mathbb{A}$. The claim follows from Theorem 4.5 with $\omega=\prod_{j} \omega_{\lambda_{j}, a_{j}}$.

Let $\boldsymbol{\omega}, v$, and $L$ be as in Theorem 4.5. Suppose that $V=W^{0}(\boldsymbol{\omega})$ and that $\boldsymbol{\omega}=$ $\prod_{j=1}^{m} \boldsymbol{\omega}_{\lambda, a_{j}}$ with $a_{j} \in \mathbb{A}$ and $a_{i} \neq a_{j}$ when $i \neq j$, so that $W^{0}(\boldsymbol{\omega}) \cong \bigotimes_{j} W^{0}\left(\boldsymbol{\omega}_{\lambda_{j}, a_{j}}\right)$; see [Chari and Pressley 2001]. Choose $\ell$-highest-weight vectors $v_{j}$ of $W\left(\omega_{\lambda_{j}, a_{j}}\right)$ such that $v=v_{1} \otimes \cdots \otimes v_{m}$, and set $L_{j}=U(\tilde{\mathfrak{g}})_{\AA} v_{j}, L^{\prime}=L_{1} \otimes_{\AA} \cdots \otimes_{\mathbb{A}} L_{m}$. As before, it follows from (1-4) that $L \subseteq L^{\prime}$.

Conjecture 4.7. $\quad$ (a) $W(\bar{\omega}) \cong L_{\mathbb{F}} . \quad$ (b) If $\bar{a}_{i} \neq \bar{a}_{j}$ for $i \neq j$, then $L=L^{\prime}$. 
Part (a) is the analogous statement of the conjecture in [Chari and Pressley 2001] mentioned in the introduction of the paper. Note Theorem 4.5 implies that $\operatorname{dim}_{\mathbb{F}}\left(L_{\mathbb{F}}\right)=\operatorname{dim}_{\mathbb{F}}\left(W^{0}(\boldsymbol{\omega})\right)$. Hence, for proving (a), it suffices to prove that $\operatorname{dim}_{\mathbb{F}}(W(\overline{\boldsymbol{\omega}})) \leq \operatorname{dim}_{\mathbb{C}}\left(W^{0}(\boldsymbol{\omega})\right)$.

Now part (b) is rather unusual since for $\mathbb{Z}$-lattices the appropriate analogous statement is false; as a counterexample, one can take $\mathfrak{g}=\mathfrak{s l}_{2}, \quad p \neq 2$, and $\boldsymbol{\omega}=$ $(1-u)(1+u)$. Below we give an example showing that equality can indeed happen when working with $\mathbb{A}$-lattices. This is actually the main point behind the choice of working with discrete valuation rings: they have plenty of units. Conjecture 4.7 has a corollary:

Corollary 4.8. Let $\lambda_{j} \in P^{+}$for $b_{j} \in \mathbb{F}^{\times}$with $j=1, \ldots k$ be such that $b_{i} \neq b_{j}$ for $i \neq j$, and $\bar{\omega}=\prod_{j} \omega_{\lambda_{j}, b_{j}}$. Then:

(a) $W(\overline{\boldsymbol{\omega}}) \cong \otimes W\left(\boldsymbol{\omega}_{\lambda_{j}, b_{j}}\right)$.

(b) If $M_{j}$ is a quotient of $W\left(\omega_{\lambda_{j}, b_{j}}\right)$, then $M=\bigotimes_{j} M_{j}$ is a quotient of $W(\overline{\boldsymbol{\omega}})$.

Proof. Let $a_{j} \in \mathbb{F}^{0}$ be such that $\bar{a}_{j}=b_{j}$. From part (a) of the conjecture we have $W(\overline{\boldsymbol{\omega}}) \cong L_{\mathbb{F}}$ and from (b) $L_{\mathbb{F}}=L_{\mathbb{F}}^{\prime}$. Now Lemma 4.4 implies $L_{\mathbb{F}}^{\prime} \cong \bigotimes_{j}\left(L_{j}\right)_{\mathbb{F}}$. Thus, applying part (a) of the conjecture to $\left(L_{j}\right)_{\mathbb{F}}$ we conclude part (a).

Once we have part (a), the proof of (b) is standard. Namely, let $V_{j}$ be the kernel of the projection $W\left(\boldsymbol{\omega}_{\lambda_{j}, b_{j}}\right) \rightarrow M_{j}$. Proceeding recursively on $j=1, \ldots, k$, we obtain short exact sequences

$$
\begin{array}{r}
0 \rightarrow\left(\bigotimes_{i=1}^{j-1} M_{i}\right) \otimes V_{j} \otimes\left(\bigotimes_{i=j+1}^{k} W\left(\boldsymbol{\omega}_{\lambda_{i}, b_{i}}\right)\right) \rightarrow\left(\bigotimes_{i=1}^{j-1} M_{i}\right) \otimes\left(\bigotimes_{i=j}^{k} W\left(\boldsymbol{\omega}_{\lambda_{i}, b_{i}}\right)\right) \\
\rightarrow\left(\bigotimes_{i=1}^{j} M_{i}\right) \otimes\left(\bigotimes_{i=j+1}^{k} W\left(\boldsymbol{\omega}_{\lambda_{i}, b_{i}}\right)\right) \rightarrow 0 .
\end{array}
$$

The characteristic zero counterpart of part (a) of the corollary was proved in [Chari and Pressley 2001, Section 3]. So far we have not managed to adapt or compliment those techniques. By transferring the problem to the setting of $\mathbb{A}$ lattices, we expect that other characteristic zero arguments, for example, as in [Feigin and Loktev 2004], will lead to a proof of Conjecture 4.7(b).

Theorem 2.2(a) leads immediately to:

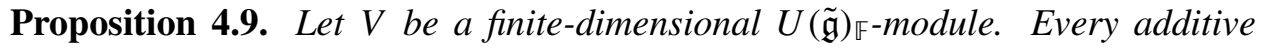
subgroup of $V$ which is invariant under the action of $U(\tilde{\mathfrak{g}})_{\mathbb{A}}$ is the direct sum of its intersection with the weight spaces of $V$.

We now give the example showing that Conjecture 4.7(b) may hold in the setting of discrete valuation rings. Let $\mathfrak{g}=\mathfrak{s l}_{2}$. Since $I$ is a singleton, we will drop the root index and write $x_{r}^{ \pm}, h_{r}$, and $\Lambda_{r}$ instead of $x_{1, r}^{ \pm}$and so on. We will also 
identify $P$ with $\mathbb{Z}$. We will verify part (b) of Conjecture 4.7 for the Weyl module $V=W^{0}\left((1-a u)^{2}(1-b u)\right)$, where $a, b \in \mathbb{A}^{\times}$for some discrete valuation ring $\mathbb{A}$ such that $\bar{a} \neq \bar{b}$. In particular, $W^{0}\left((1-a u)^{2}(1-b u)\right) \cong W^{0}\left((1-a u)^{2}\right) \otimes W^{0}(1-b u)$. Let $v_{0}$ and $w_{0}$ be $\ell$-highest weight vectors of $W^{0}\left((1-a u)^{2}\right)$ and $W^{0}(1-b u)$, respectively.

$W^{0}(1-b u)$ is isomorphic to the evaluation representation $V^{0}(1, b)$. It is then easy to see that $x_{s}^{-} w_{0}=b^{s} x_{0}^{-} w_{0}$ for all $s \in \mathbb{Z}$. Thus, letting $w_{1}=x_{0}^{-} w_{0}$, the set $\left\{w_{0}, w_{1}\right\}$ is an $\mathbb{A}$-basis for $L_{2}=U(\tilde{\mathfrak{g}})_{\AA} w_{0}$.

Now consider $W^{0}\left((1-a u)^{2}\right)$ and let $L_{1}=U(\tilde{\mathfrak{g}})_{\AA} v_{0}$. Since $\operatorname{wt}\left((1-a u)^{2}\right)=2$, letting $k>2$ in (1-11) we get

$$
\left(\left(X_{\alpha ; s,+}^{-}(u)\right)^{(k-l)} \Lambda_{\alpha}^{+}(u)\right)_{k} v_{0}=0 \quad \text { for all } l, s \in \mathbb{Z} \text { with } 1 \leq l \leq k .
$$

Setting $k=3$ and $l=2$ above, we get $\left(x_{s+1}^{-} \Lambda_{2}+x_{s+2}^{-} \Lambda_{1}+x_{s+3}^{-}\right) v_{0}=0$. Since $\Lambda_{2} v_{0}=a^{2} v_{0}$ and $\Lambda_{1} v_{0}=-2 a v_{0}$, one easily proves inductively that

$$
x_{s}^{-} v_{0}=s a^{s-1} x_{1}^{-} v_{0}-(s-1) a^{s} x_{0}^{-} v_{0} \quad \text { for all } s \in \mathbb{Z} .
$$

Let $v_{1}=x_{0}^{-} v_{0}$ and $v_{3}=x_{1}^{-} v_{0}$. Thus we see that $\left\{v_{1}, v_{3}\right\}$ is an $\mathbb{A}$-basis for the zero-weight space of $W^{0}\left((1-a u)^{2}\right) \cap L_{1}$. Now setting $k=3, l=1$ in (4-20), we get $\left(x_{s+1}^{-}\right)^{(2)} \Lambda_{1} v_{0}+x_{s+1}^{-} x_{s+2}^{-} v_{0}=0$. Setting $s=-1$ we get

$$
x_{1}^{-} x_{0}^{-} v_{0}=2 a\left(x_{0}^{-}\right)^{(2)} v_{0},
$$

and setting $s=0$ we get $2 a\left(x_{1}^{-}\right)^{(2)} v_{0}=x_{1}^{-} x_{2}^{-} v_{0}$. Now using (4-21) and then (4-22) on the right hand side of the last equation gives

$$
\left(x_{1}^{-}\right)^{(2)} v_{0}=a^{2}\left(x_{0}^{-}\right)^{(2)} v_{0} .
$$

Finally, using (4-21), (4-22), and (4-23) we get

$$
x_{r}^{-} x_{s}^{-} v_{0}=2 a^{r+s}\left(x_{0}^{-}\right)^{(2)} v_{0} \quad \text { for all } r, s \in \mathbb{Z} .
$$

Hence $v_{2}=\left(x_{0}^{-}\right)^{(2)} v_{0}$ completes an $\mathbb{A}$-basis for $L_{1}$, that is, $L_{1}$ is the $\mathbb{A}$-span of $\left\{v_{0}, v_{1}, v_{2}, v_{3}\right\}$.

Clearly the set $A=\left\{v_{i} \otimes w_{j}: i=0,1,2,3\right.$ and $\left.j=0,1\right\}$ is an $\mathbb{A}$-basis for $L^{\prime}=L_{1} \otimes_{\mathbb{A}} L_{2}$. Since $L=U(\tilde{\mathfrak{g}})_{\mathbb{A}}\left(v_{0} \otimes w_{0}\right) \subseteq L^{\prime}$, we are left to show that $A \subseteq L$. Using (4-21), (4-24) and $x_{s}^{-} w_{0}=b^{s} x_{0}^{-} w_{0}$ we compute

$$
\begin{aligned}
& x_{0}^{-}\left(v_{0} \otimes w_{0}\right)=v_{1} \otimes w_{0}+v_{0} \otimes w_{1}, \\
& x_{1}^{-}\left(v_{0} \otimes w_{0}\right)=v_{3} \otimes w_{0}+b v_{0} \otimes w_{1}, \\
& x_{2}^{-}\left(v_{0} \otimes w_{0}\right)=2 a v_{3} \otimes w_{0}-a^{2} v_{1} \otimes w_{0}+b^{2} v_{0} \otimes w_{1} .
\end{aligned}
$$


Recording the coordinates of these vectors in the basis $\left\{v_{1} \otimes w_{0}, v_{3} \otimes w_{0}, v_{0} \otimes w_{1}\right\}$ of $L^{\prime} \cap V_{1}$ we get the matrix

$$
\left[\begin{array}{ccc}
1 & 0 & -a^{2} \\
0 & 1 & 2 a \\
1 & b & b^{2}
\end{array}\right],
$$

whose determinant is $(a-b)^{2}$. Since $\bar{a} \neq \bar{b}$ if and only if $a-b \in \mathbb{A}^{\times}$, we see that the vectors $x_{0}^{-}\left(v_{0} \otimes w_{0}\right), x_{1}^{-}\left(v_{0} \otimes w_{0}\right)$, and $x_{2}^{-}\left(v_{0} \otimes w_{0}\right)$ also form an $\mathbb{A}$-basis for $L^{\prime} \cap V_{1}$. Now we compute

$$
\begin{aligned}
\left(x_{0}^{-}\right)^{(2)}\left(v_{0} \otimes w_{0}\right) & =v_{2} \otimes w_{0}+v_{1} \otimes w_{1}, \\
x_{1}^{-} x_{0}^{-}\left(v_{0} \otimes w_{0}\right) & =2 a v_{2} \otimes w_{0}+b v_{1} \otimes w_{1}+v_{3} \otimes w_{1}, \\
\left(x_{1}^{-}\right)^{(2)}\left(v_{0} \otimes w_{0}\right) & =a^{2} v_{2} \otimes w_{0}+b v_{3} \otimes w_{1},
\end{aligned}
$$

and, in the basis $\left\{v_{2} \otimes w_{0}, v_{1} \otimes w_{1}, v_{3} \otimes w_{1}\right\}$ of $L^{\prime} \cap V_{-1}$, these vectors have the matrix

$$
\left[\begin{array}{ccc}
1 & 2 a & a^{2} \\
1 & b & 0 \\
0 & 1 & b
\end{array}\right] .
$$

The determinant is again $(a-b)^{2}$ and we are done with this weight space as before. Finally, one easily sees that $\left(x_{0}^{-}\right)^{(3)}\left(v_{0} \otimes w_{0}\right)=v_{2} \otimes w_{1}$, showing that $A \subseteq L$ as claimed.

Let us end this subsection proving the existence of the automorphisms $\psi_{a}$, as promised at the end of Proposition 4.2.

Proposition 4.10. For every $a \in \mathbb{F}^{\times}$there exists an algebra automorphism $\psi_{a}$ : $U(\tilde{\mathfrak{g}})_{\mathbb{F}} \rightarrow U(\tilde{\mathfrak{g}})_{\mathbb{F}}$ sending $\left(x_{\alpha, r}^{ \pm}\right)^{(k)}$ to $a^{r k}\left(x_{\alpha, r}^{ \pm}\right)^{(k)}$.

Proof. Let $b$ be a lift of $a$ to $\mathbb{A}$, and let $\psi_{b}^{0}: U(\tilde{\mathfrak{g}})_{\mathbb{F}^{0}} \rightarrow U(\tilde{\mathfrak{g}})_{\mathbb{F}^{0}}$ be the algebra automorphism extending $t \mapsto b t$. It is easy to see that $\psi_{b}^{0}$ maps $U(\tilde{\mathfrak{g}})_{\AA}$ onto itself. Now let $\psi_{a}$ be the reduction modulo $p$ of the restriction of $\psi_{b}^{0}$ to $U(\tilde{\mathfrak{g}})_{A}$.

Remark. The same kind of argument gives an alternate proof of Proposition 3.3 without using the formal evaluation map but using instead the $\mathbb{A}$-form $U(\tilde{\mathfrak{g}})_{\mathbb{A}}$.

4D. Block decomposition. We now assume Conjecture 4.7 to obtain the block decomposition of the category of finite-dimensional $U(\tilde{\mathfrak{g}})_{\mathbb{F}}$-modules. We begin with a proposition on the Jordan-Hölder series of Weyl modules.

Proposition 4.11. The $\ell$-weights of $W\left(\omega_{\lambda, a}\right)$ are of the form $\omega_{\mu, a}$ with $\mu \in P$ such that $\mu \leq \lambda$.

Proof. Let $b$ be a lift of $a$ to $\mathbb{A}$, consider $W^{0}\left(\boldsymbol{\omega}_{\lambda, b}\right)$, and let $L=U(\tilde{\mathfrak{g}})_{\mathbb{F}} v$ for some choice of $\ell$-highest-weight vector $v$ of $W^{0}\left(\boldsymbol{\omega}_{\lambda, b}\right)$. It is well known that the $\ell$-weights of $W^{0}\left(\boldsymbol{\omega}_{\lambda, b}\right)$ are of the form $\boldsymbol{\omega}_{\mu, b}$ with $\mu \in P$ such that $\mu \leq \lambda$ (see [Chari 
and Moura 2004, Proposition 3.3] and [Chari and Moura 2005] for instance). In particular, the weight spaces of $W^{0}\left(\boldsymbol{\omega}_{\lambda, b}\right)$ coincide with its $\ell$-weight spaces, and therefore, using Proposition 4.9, we conclude that $L$ is equal to its intersection with the $\ell$-weight spaces of $W^{0}\left(\boldsymbol{\omega}_{\lambda, b}\right)$. Since by Conjecture $4.7(a) W\left(\boldsymbol{\omega}_{\lambda, a}\right)$ is isomorphic to $L_{\mathbb{E}}$, the proposition is now easily deduced.

For each $a \in \mathbb{F}^{\times}$and $i \in I$, set $\omega_{i, a}=\omega_{\omega_{i}, a}$ (the $\ell$-fundamental weights) and $\alpha_{i, a}(u)=\omega_{\alpha_{i}, a}$ (the $\ell$-simple roots). Let $\mathscr{Q}_{\mathbb{F}}$ be the subgroup of $\mathscr{P}_{\mathbb{F}}$ generated by all $\alpha_{i, a}(u)$; similarly, let $\mathscr{Q}_{\mathbb{F}}^{+}$be the submonoid of $\mathscr{P}_{\mathbb{F}}$ generated by all $\alpha_{i, a}(u)$. We call $\mathscr{Q}_{\mathbb{E}}$ the $\ell$-root lattice. The last proposition and Corollary 4.8 will give

Corollary 4.12. If $V$ is a finite-dimensional $\ell$-highest-weight $U(\tilde{\mathfrak{g}})_{\mathbb{F}}$-module with $\ell$-highest weight $\omega$, then $V_{\varpi} \neq 0$ only if $\varpi \in \omega\left(2_{\mathbb{F}}^{+}\right)^{-1}$.

Proof. Proposition 4.11 implies the result holds for $W\left(\boldsymbol{\omega}_{\lambda, a}\right)$. Then Corollary 4.8 finishes the proof. In fact, (1-5) implies that the $\ell$-weights of the tensor product are products of the $\ell$-weights of each tensor factor; see [Chari and Moura 2005, Lemma 4.4].

Definition 4.13. A spectral character is a function $\chi: \mathbb{F}^{\times} \rightarrow P / Q$ with finite support. Equipping the space of all spectral characters $\Xi_{\mathbb{F}}$ with the usual abelian group structure, one sees that the assignment $\omega_{i, a} \mapsto \chi_{i, a}$, where $\chi_{i, a}(b)=\delta_{a, b} \omega_{i}$, determines a group homomorphism $\mathscr{P}_{\mathbb{F}} \rightarrow \Xi_{\mathbb{F}}, \varpi \mapsto \chi_{\varpi}$, with kernel $\mathscr{Q}_{\mathbb{F}}$. We say a $U(\tilde{\mathfrak{g}})_{\mathbb{F}}$-module $V$ has spectral character $\chi$ if $\chi \varpi=\chi$ whenever $V_{\varpi} \neq 0$. Let $\widetilde{\mathscr{C}}_{\chi}$ be the category of all finite-dimensional $U(\tilde{\mathfrak{g}})_{\mathbb{F}}$-modules with spectral character $\chi$.

We will denote by $\chi_{\mu, a}$ the spectral character corresponding to $\omega_{\mu, a}$ for $\mu \in P$ and $a \in \mathbb{F}^{\times}$. We use additive notation for the group operation of $\Xi_{\mathbb{F}}$.

Proposition 4.14. (a) For all $\omega \in \mathscr{P}_{\mathbb{F}}^{+}, W(\omega) \in \tilde{\mathscr{C}}_{\chi \omega}$.

(b) $\widetilde{\mathscr{C}}_{\chi_{1}} \otimes \widetilde{\mathscr{C}}_{\chi_{2}} \subseteq \widetilde{\mathscr{C}}_{\chi_{1}+\chi_{2}}$ for all $\chi_{1}, \chi_{2} \in \Xi_{\mathbb{F}}$.

(c) If $V \in \widetilde{\mathscr{C}}_{\chi}$ then $V^{*} \in \widetilde{\mathscr{C}}_{-\chi}$.

Proof. Parts (a) and (b) are immediate from Corollary 4.12 and its proof. Part (c) follows from Proposition 3.7.

Let $\widetilde{\mathscr{C}}_{\mathbb{F}}$ be the category of all finite-dimensional $U(\tilde{\mathfrak{g}})_{\mathbb{F}}$-modules. In the rest of the section we prove that the block decomposition of $\widetilde{\mathscr{C}}_{\mathbb{F}}$ is described just as in the characteristic zero case [Chari and Moura 2004] and quantum group case [Chari and Moura 2005; Etingof and Moura 2003]. Namely:

Theorem 4.15. The categories $\widetilde{\mathscr{C}}_{\chi}$ for $\chi \in \Xi_{\mathbb{F}}$ are the blocks of $\widetilde{\mathscr{C}}_{\mathbb{F}}$. 
Once we have the statements of Propositions 4.14 and 2.9 available, arguments identical to those [Chari and Moura 2004, Section 5] show that every indecomposable object from $\widetilde{\mathscr{C}}_{\mathbb{F}}$ belongs to some $\widetilde{\mathscr{C}}_{\chi}$, proving that we have the decomposition

$$
\tilde{\mathscr{C}}_{\mathbb{F}}=\bigoplus_{\chi \in \Xi_{\mathbb{F}}} \tilde{\mathscr{C}}_{\chi} \text {. }
$$

It remains to see that $\widetilde{\mathscr{C}}_{\chi}$ are indecomposable abelian subcategories. To do this, it suffices to show that for any two given irreducible $U(\tilde{\mathfrak{g}})_{\mathbb{F}}$-modules $V$ and $W$ having the same spectral character, there exists a finite sequence of indecomposable objects $M_{1}, \ldots, M_{k}$ such that $V$ is a simple constituent of $M_{1}, W$ is a simple constituent of $M_{k}$, and for every $j, M_{j}$ has a common simple constituent with $M_{j+1}$; see [Etingof and Moura 2003, Section 1]. Let us begin with the case when $V=V(\lambda, a)$ and $W=V(\mu, b)$ for some $\lambda, \mu \in P^{+}$and $a, b \in \mathbb{F}^{\times}$. Quite clearly $\chi_{\lambda, a}=\chi_{\mu, b}$ if and only if $\lambda-\mu \in Q$, and if $\lambda \notin Q$, also $a=b$.

Proposition 4.16. Let $a \in \mathbb{F}^{\times}$, and suppose $\lambda, \mu \in P^{+}$are such that $\operatorname{Hom}_{\mathfrak{g}_{\mathbb{F}_{0}}}\left(\mathfrak{g}_{\mathbb{F}_{0}} \otimes\right.$ $\left.V^{0}(\lambda), V^{0}(\mu)\right) \neq 0$ and $\lambda>\mu$. Then there exists a quotient $M$ of $W\left(\omega_{\lambda, a}\right)$ having $V(\mu, a)$ as simple constituent.

Proof. Let $b$ be a lift of $a$ to A. By [Chari and Moura 2004, Proposition 3.4], there exists a nonsplit short exact sequence of $\tilde{\mathfrak{g}}_{\mathfrak{F}_{0}}$-modules:

$$
0 \rightarrow V^{0}(\mu, b) \rightarrow M^{0} \rightarrow V^{0}(\lambda, b) \rightarrow 0
$$

for some $\ell$-highest-weight module $M^{0}$. From Theorem 4.5, there exists an admissible ample-lattice $L$ for $M^{0}$ such that $M=L_{\mathbb{F}}$ is a quotient of $W\left(\omega_{\lambda, a}\right)$. It remains to show that there exists an $\ell$-highest-weight vector $v^{\prime}$ for $V^{0}(\mu, b)$ in $M^{0}$ such $v^{\prime} \in L$ and its image in $L_{\mathbb{F}}$ is nonzero. Thus, let $v$ be an $\ell$-highest-weight vector for $M^{0}$. From the proof of Theorem 3.11, using that $b \in \mathbb{A}^{\times}$as in the proof of Proposition 4.2, we see that there exists an A-basis for $L$ consisting of vectors that are $\mathbb{A}$-linear combinations of elements of the form $\left(x_{\alpha_{1}, r_{1}}^{-}\right)^{\left(k_{1}\right)} \cdots\left(x_{\alpha_{m}, r_{m}}^{-}\right)^{\left(k_{m}\right)} v$. Let $v_{1}, \ldots, v_{n}$ be an $\mathbb{A}$-basis for $L_{\mu}$. Any $\ell$-highest-weight vector for $V^{0}(\mu, b)$ is a solution $\sum_{j=1}^{n} c_{j} v_{j}$ for some $c_{j} \in \mathbb{F}^{0}$ of the linear system

$$
\left(x_{\alpha, r}^{+}\right)^{(k)} \sum_{j=1}^{n} c_{j} v_{j}=0
$$

for all $\alpha \in R^{+}, r \in \mathbb{Z}$ and $k \in \mathbb{Z}_{+}$. Since $L$ is admissible and the $\ell$-weights of $M^{0}$ are in $\mathscr{P}_{\mathrm{A}}$ (Proposition 4.11), it follows that there exists a solution with the $c_{j}$ lying in the field of fractions of $\mathbb{A}$. Since $\mathbb{A}$ is a unique factorization domain, it follows that we can choose $c_{j}$ in $\mathbb{A}$ such that the nonzero $c_{j}$ are coprime.

This proposition and Corollary 4.8(b) imply: 
Corollary 4.17. Let $a=a_{0}, \lambda, \mu, M$ be as in Proposition 4.16, and for $j=$ $1, \ldots, k$, let $v_{j} \in P^{+}$and $a_{j} \in \mathbb{F}^{\times}$be such that $a_{i} \neq a_{l}$ for all $i, l=0, \ldots, k$ with $i \neq l$. Then the $U(\tilde{\mathfrak{g}})_{\mathbb{E}}$-module $M \otimes\left(\otimes_{j} V\left(v_{j}, a_{j}\right)\right)$ is $\ell$-highest-weight and has $V(\mu, a) \otimes\left(\bigotimes_{j} V\left(v_{j}, a_{j}\right)\right)$ and $V(\lambda, a) \otimes\left(\bigotimes_{j} V\left(v_{j}, a_{j}\right)\right)$ as simple constituents.

Now let $a \in \mathbb{F}^{\times}$and $\lambda, \mu \in P^{+}$be such that $\lambda-\mu \in Q-\{0\}$. Then by [Chari and Moura 2004, Proposition 1.2], there exists a finite sequence $\lambda=v_{1}, v_{2}, \ldots, v_{k}=\mu$ such that $v_{j} \neq v_{j+1}$ and $\operatorname{Hom}_{\mathfrak{g}_{\mathbb{F}_{0}}}\left(\mathfrak{g}_{\mathbb{F}_{0}} \otimes V^{0}\left(v_{j}\right), V^{0}\left(v_{j+1}\right)\right) \neq 0$. Since

$$
\operatorname{Hom}_{\mathfrak{g}_{\digamma_{0}}}\left(\mathfrak{g}_{\mathbb{F}_{0}} \otimes V^{0}\left(v_{j}\right), V^{0}\left(v_{j+1}\right)\right)=\operatorname{Hom}_{\mathfrak{g}_{\digamma_{0}}}\left(\mathfrak{g}_{\mathbb{F}_{0}} \otimes V^{0}\left(v_{j+1}\right), V^{0}\left(v_{j}\right)\right),
$$

we conclude that there exists a sequence of $U(\tilde{\mathfrak{g}})_{\mathbb{F}}-\ell$-highest-weight modules $M_{j}$ for $j=1, \ldots, k-1$ having both $V\left(v_{j}, a\right)$ and $V\left(v_{j+1}, a\right)$ as simple constituents. From here it is quite clear how to complete the proof of Theorem 4.15 using the last corollary; see [Chari and Moura 2004, Section 4].

Remark. We give an informal argument to justify why it should be expected that the block decomposition of $\widetilde{\mathscr{C}}_{\mathbb{F}}$ is described like that of $\widetilde{\mathscr{C}}_{\mathbb{F}_{0}}$, contrary to what happens with the block decompositions of $\mathscr{C}_{\mathbb{F}}$ and $\mathscr{C}_{\mathbb{F}_{0}}$ (the categories of finitedimensional representations for $U(\mathfrak{g})_{\mathbb{F}}$ and $U(\mathfrak{g})_{F_{0}}$ ). While the blocks of $\mathscr{C}_{\mathbb{F}_{0}}$ are as small as possible $\left(\mathscr{C}_{\mathbb{F}_{0}}\right.$ is a semisimple category), the blocks of $\tilde{\mathscr{C}}_{\mathbb{F}_{0}}$ are as large as one can expect (for instance, when $P / Q$ is trivial, $\widetilde{\mathscr{C}}_{\mathbb{F}_{0}}$ is itself an indecomposable abelian category). Hence, while the blocks of $\mathscr{C}_{\mathbb{F}}$ have space to become "larger" and they indeed become so, but still not as large as possible [Jantzen 1987, Chapter II.7] - that is not the case for $\widetilde{\mathscr{C}}_{\mathbb{F}}$.

\section{Acknowledgements}

Jakelić thanks the Max-Planck-Institut für Mathematik in Bonn for its hospitality and support, FAPESP (processo 2006/00609-1) for supporting her visit to the University of Campinas when part of this paper was developed, and UNICAMP for its hospitality. Moura's research is partially supported by $\mathrm{CNPq}$ (processo 303349/2005-0) and FAPESP (processo 2006/00833-9). We thank V. Chari for turning our attention to this subject and for helpful questions and suggestions. We also thank P. Russel, A. Engler, and P. Brumatti for useful discussions and pointers about discrete valuation rings and L. Scott for his interest and helpful references.

\section{References}

[Beck and Nakajima 2004] J. Beck and H. Nakajima, "Crystal bases and two-sided cells of quantum affine algebras”, Duke Math. J. 123:2 (2004), 335-402. MR 2005e:17020 Zbl 1062.17006

[Borel et al. 1970] A. Borel, R. Carter, C. Curtis, N. Iwahori, T. Springer, and R. Steinberg, Seminar on Algebraic Groups and Related Finite Groups (Institute for Advanced Study, Princeton, 
NJ, 1968/69), vol. 131, Lecture Notes in Mathematics, Springer, Berlin, 1970. MR 0258840 Zbl 0192.36201

[Chari 1986] V. Chari, "Integrable representations of affine Lie-algebras”, Invent. Math. 85:2 (1986), 317-335. MR 88a:17034 Zbl 0603.17011

[Chari and Greenstein 2007] V. Chari and J. Greenstein, "Graded level zero representations of affine Lie algebras", Trans. Am. Math. Soc. (2007). To appear.

[Chari and Loktev 2006] V. Chari and S. Loktev, "Weyl, Demazure and fusion modules for the current algebra of $\mathfrak{s l}_{r+1}$ ", Adv. Math. 207:2 (2006), 928-960. MR 2008a:17029 Zbl 05077981

[Chari and Moura 2004] V. Chari and A. A. Moura, "Spectral characters of finite-dimensional representations of affine algebras”, J. Algebra 279:2 (2004), 820-839. MR 2005f:17002 Zbl 02104467

[Chari and Moura 2005] V. Chari and A. A. Moura, "Characters and blocks for finite-dimensional representations of quantum affine algebras", Int. Math. Res. Not. 5 (2005), 257-298. MR 2006a: $17021 \mathrm{Zbl} 1074.17004$

[Chari and Pressley 1986] V. Chari and A. Pressley, "New unitary representations of loop groups", Math. Ann. 275:1 (1986), 87-104. MR 88f:17029 Zbl 0603.17012

[Chari and Pressley 1997] V. Chari and A. Pressley, "Quantum affine algebras at roots of unity", Represent. Theory 1 (1997), 280-328. MR 98e:17018 Zbl 0891.17013

[Chari and Pressley 2001] V. Chari and A. Pressley, "Weyl modules for classical and quantum affine algebras", Represent. Theory 5 (2001), 191-223. MR 2002g:17027 Zbl 0989.17019

[Cline et al. 1980] E. Cline, B. Parshall, and L. Scott, "On the tensor product theorem for algebraic groups”, J. Algebra 63:1 (1980), 264-267. MR 81e:20046 Zbl 0428.20024

[Cline et al. 2000] E. Cline, B. Parshall, and L. Scott, "Witt vectors and affine Lie algebras", pp. 57-87 in Representations and quantizations (Shanghai, 1998), edited by J. Wang and Z. Lin, China High. Educ. Press, Beijing, 2000. MR 2002b:17024 Zbl 1002.17012

[Etingof and Moura 2003] P. I. Etingof and A. A. Moura, "Elliptic central characters and blocks of finite dimensional representations of quantum affine algebras", Represent. Theory 7 (2003), 346373. MR 2004j:17017 Zbl 1066.17005

[Feigin and Loktev 2004] B. Feigin and S. Loktev, "Multi-dimensional Weyl modules and symmetric functions", Comm. Math. Phys. 251:3 (2004), 427-445. MR 2005m:17005 Zbl 1100.17005

[Fourier and Littelmann 2006] G. Fourier and P. Littelmann, "Tensor product structure of affine Demazure modules and limit constructions", Nagoya Math. J. 182 (2006), 171-198. MR 2007e:17021

[Fourier and Littelmann 2007] G. Fourier and P. Littelmann, "Weyl modules, Demazure modules, KR-modules, crystals, fusion products and limit constructions", Adv. Math. 211:2 (2007), 566-593. MR 2323538 Zbl 1114.22010

[Garland 1978] H. Garland, "The arithmetic theory of loop algebras”, J. Algebra 53:2 (1978), 480551. MR 80a:17012 Zbl 0383.17012

[Garland 1980] H. Garland, "The arithmetic theory of loop groups", Inst. Hautes Études Sci. Publ. Math. 52 (1980), 5-136. MR 83a:20057 Zbl 0475.17004

[Humphreys 1972] J. Humphreys, Introduction to Lie algebras and representation theory, Springer, New York, 1972. Graduate Texts in Mathematics, Vol. 9. MR 48 \#2197 Zbl 0254.17004

[Humphreys 1977] J. Humphreys, "On the hyperalgebra of a semisimple algebraic group", pp. 203210 in Contributions to algebra (collection of papers dedicated to Ellis Kolchin), Academic Press, New York, 1977. MR 57 \#6211 Zbl 0367.20043

[Jantzen 1987] J. C. Jantzen, Representations of algebraic groups, Pure and Applied Mathematics 131, Academic Press, Boston, 1987. MR 89c:20001 Zbl 0654.20039

[Kashiwara 1994] M. Kashiwara, "Crystal bases of modified quantized enveloping algebra", Duke Math. J. 73:2 (1994), 383-413. MR 95c:17024 Zbl 0794.17009 
[Kashiwara 2002] M. Kashiwara, "On level-zero representations of quantized affine algebras", Duke Math. J. 112:1 (2002), 117-175. MR 2002m:17013 Zbl 1033.17017

[Kostant 1966] B. Kostant, "Groups over Z”, pp. 90-98 in Algebraic Groups and Discontinuous Subgroups (Boulder, CO, 1965), edited by A. Borel and G. D. Mostow, Amer. Math. Soc., Providence, R.I., 1966. MR 34 \#7528 Zbl 0199.06903

[Kuniba et al. 1998] A. Kuniba, K. C. Misra, M. Okado, T. Takagi, and J. Uchiyama, "Crystals for Demazure modules of classical affine Lie algebras", J. Algebra 208:1 (1998), 185-215. MR 99h:17008 Zbl 0974.17028

[Kuniba et al. 2000] A. Kuniba, K. C. Misra, M. Okado, T. Takagi, and J. Uchiyama, "Paths, Demazure crystals, and symmetric functions", J. Math. Phys. 41 (2000), 6477-6486. MR 2002f:17024 Zbl 0971.17006

[Mathieu 1988] O. Mathieu, "Formules de caractères pour les algèbres de Kac-Moody générales", Astérisque 159-160 (1988), 267. MR 90d:17024 Zbl 0683.17010

[Mathieu 1996] O. Mathieu, "On some modular representations of affine Kac-Moody algebras at the critical level”, Compositio Math. 102:3 (1996), 305-312. MR 97g:17026 Zbl 0878.17023

[Mitzman 1985] D. Mitzman, Integral bases for affine Lie algebras and their universal enveloping algebras, Contemporary Mathematics 40, American Mathematical Society, Providence, RI, 1985. MR 86h:17022 Zbl 0572.17009

[Nakajima 2001] H. Nakajima, "Quiver varieties and finite-dimensional representations of quantum affine algebras”, J. Amer. Math. Soc. 14:1 (2001), 145-238. MR 2002i:17023 Zbl 0981.17016

[Nakajima 2004] H. Nakajima, "Extremal weight modules of quantum affine algebras", pp. 343-369 in Representation theory of algebraic groups and quantum groups, edited by T. Shoji et al., Adv. Stud. Pure Math. 40, Math. Soc. Japan, Tokyo, 2004. MR 2005g:17036 Zbl 1088.17008

[Serre 1979] J.-P. Serre, Local fields, Graduate Texts in Mathematics 67, Springer, New York, 1979. MR 82e:12016 Zbl 0423.12016

[Steinberg 1963] R. Steinberg, "Representations of algebraic groups", Nagoya Math. J. 22 (1963), 33-56. MR 27 \#5870 Zbl 0271.20019

[Sullivan 1978] J. B. Sullivan, "Simply connected groups, the hyperalgebra, and Verma's conjecture", Amer. J. Math. 100:5 (1978), 1015-1019. MR 80a:14022 Zbl 0415.14028

[Tits 1980-1981] J. Tits, Résumé de cours, Annuaire du Collège de France, 1980-1981.

[Tits 1987] J. Tits, "Uniqueness and presentation of Kac-Moody groups over fields", J. Algebra 105:2 (1987), 542-573. MR 89b:17020 Zbl 0626.22013

Received December 11, 2006. Revised September 11, 2007.

\author{
DIJANA JAKELIĆ \\ MAX-PlanCK-InSTITUT FÜR MATHEMATIK \\ D-53111 BONN \\ GERMANY \\ jakelic@mpim-bonn.mpg.de
}

\author{
ADRIANO ADREGA DE MOURA \\ UNICAMP - IMECC \\ 13083-970 CAMPINAS, SP \\ BRAZIL \\ aamoura@ime.unicamp.br \\ www.ime.unicamp.br/ aamoura
}

